\title{
Susceptibilidad del suelo al impacto humano: caso del herbicida atrazina
}

\author{
Maricarmen Salazar-Ledesma, Lucy Mora, Bruno Chávez, Daniel Gómez, Olivia Zamora, \\ Blanca Prado
}

Maricarmen Salazar-Ledesma

Posgrado en Ciencias de la Tierra, Instituto de Geología, UNAM, Ciudad Universitaria, C.P. 04510, Ciudad de México, México.

\section{Lucy Mora}

Bruno Ghávez

Olivia Zamora

Departamento de Edafología, Instituto de Geología, Universidad Nacional Autónoma de México, Ciudad Universitaria, C.P. 04510, Ciudad de México, México.

Laboratorio Nacional de Geoquímica y Mineralogía (LANGEM), Universidad Nacional Autónoma de México, Ciudad Universitaria, C.P. 04510, Ciudad de México, México.

\section{Daniel Gómez}

Facultad de Química, Universidad Nacional Autónoma de México, Ciudad Universitaria, C.P. 04510, Ciudad de México, México.

\section{Blanca Prado}

bprado@geologia.unam.mx

Departamento de Edafología, Instituto de Geología, Universidad Nacional Autónoma de México, Ciudad Universitaria, C.P. 04510, Ciudad de México, México.

BOL. SOC. GEOL. MEX. 2018

VOL. 70 NO. 1

P. $95-119$

http://dx.doi.org/10.18268/BSGM2018v70nla6

\section{RESUMEN}

El uso inadecuado de sustancias químicas como los herbicidas, así como las prácticas de manejo agrícola, son sólo algunas de las actividades humanas que han provocado el deterioro del recurso suelo durante el Antropoceno. La atrazina es el herbicida más utilizado en el cultivo de maíz (Zea mays) alrededor del mundo, ya que mejora de forma importante los rendimientos agrícolas, sin embargo, también constituye un factor de riesgo de contaminación ambiental y para la salud humana. El objetivo de este trabajo fue evaluar la capacidad de adsorción - desorción del herbicida atrazina en tres tipos de suelo bajo diferente manejo agrícola: (i) Andosol, agricultura de temporal; (ii) Feozem, labranza convencional (LC) y labranza cero (LZ); y (iii) Vertisol, de temporal (VT), y con diferente número de años bajo riego con agua residual (V56 y V100). Se realizaron experimentos estándar en lote en muestras de suelo con y sin materia orgánica (MO) para cada tipo de suelo y se obtuvieron los coeficientes de distribución $\left(\mathrm{K}_{\mathrm{d}}\right)$ correspondientes. La capacidad de adsorción fue mayor en las muestras de suelo con MO, obteniéndose el valor más alto en el Andosol (19 L/ kg), después en el Vertisol (3 a $11 \mathrm{~L} / \mathrm{kg}$ ) y el más bajo en el Feozem $(1 \mathrm{~L} / \mathrm{kg})$. Sin embargo, la mayor afinidad del herbicida por la fracción orgánica del suelo, no sólo está relacionada con su contenido, sino también con su composición química (grado de aromaticidad), misma que depende en gran medida de las prácticas de manejo agrícola de cada tipo de suelo. Actividades como: la rotación de cultivos, la incorporación de los residuos al final del ciclo de cultivo, el uso de agua residual para riego y el tipo de labranza realizada, son factores que inciden en la calidad de la MO del suelo y por lo tanto en la retención de atrazina, lo cual contribuye en la función amortiguadora del suelo y en el destino ambiental del herbicida.

Palabras clave: Atrazina, adsorción-desorción, materia orgánica del suelo, manejo agrícola, Andosol, Feozem, Vertisol.

\section{ABSTRACT}

Soil deterioration during the Anthropocene is a result of human activities such as misuse of chemical compounds like herbicides and inadequate agricultural management practices. Atrazine is the most widely used herbicide in maize (Zea mays) crops around the world. Its application is used for improving agricultural yields. However, it also represents a risk factor towards environmental contamination and human health. The objective of this work was to evaluate the adsorption-desorption capacity of atrazine in three types of soils under different agricultural management: (i) Andosol under rainfed agriculture; (ii) Phaeozem under conventional tillage (LC) and under zero tillage (LZ); and (iii) Vertisol under rainfed agriculture (VT), and Vertisols irrigated with raw wastewater during 56 and 100 years (V56 and V100). Standard batch experiments were performed on samples of each soil type with and without organic matter (OM). Adsorption capacity was higher in the soil samples containing $O M$. The following distribution coefficients $(K d)$ were obtained: the highest $K_{d}$ value was obtained in the Andosol (19 L/kg), followed by the Vertisol (3 to $11 \mathrm{~L} / \mathrm{kg})$, and the lowest was in the Phaeozem $(1 \mathrm{~L} / \mathrm{kg})$. We found that the larger affinity of the herbicide for the organic fraction of the soil is not only related to the organic content, but also due to its chemical composition (degree of aromaticity). This depends mainly upon the agricultural management practices such as crop rotation and the incorporation of residues at the end of the crop cycle. Also, the use of wastewater during irrigation and the type of tillage performed are additional factors that affect the quality of soil organic matter. Therefore, the atrazine retention capacity of the soil, contributes to the soil buffering function and to the environmental destination of the herbicide.

Keyzords: Atrazine, adsorption-desorption, soil organic matter, agricultural management, Andosol, Phaeozem, Vertisol. 


\section{Introducción}

Hablando del suelo, el Antropoceno puede ser descrito como una época de degradación del recurso, notablemente desde principios del siglo XVIII (Blum y Eswaran, 2004). Las principales amenazas para el deterioro del suelo son: la erosión, el sellamiento, la reducción de la biodiversidad edáfica, la salinización, los deslizamientos de terreno, la disminución del contenido de materia orgánica (MO) y la contaminación local y difusa del suelo. En esta última se ubica el manejo inadecuado de sustancias que forman parte de prácticas agrícolas (COM, 2002).

La demanda mundial de alimentos ha aumentado en el orden del $2 \%$ por año como respuesta al crecimiento demográfico en los últimos 50 años (FAO, 2011). Por lo tanto, la velocidad a la cual se deben producir los alimentos promueve el agotamiento de los recursos, es decir, el suelo no tiene el tiempo suficiente para recuperar el nivel de nutrientes de forma natural (Salazar-López y Aldana-Madrid, 2011), de tal forma que el uso de agroquímicos forma parte de las prácticas comunes en la agricultura moderna. Grandes cantidades de agroquímicos son aplicadas a los campos agrícolas con el propósito de mejorar el rendimiento de los cultivos (fertilizantes), así como también para controlar malezas (herbicidas), hongos patógenos de plantas (fungicidas) e insectos nocivos (insecticidas). Entre los agroquímicos, los plaguicidas constituyen el grupo de productos químicos industriales más utilizados en la actualidad, de los cuales los herbicidas son los más utilizados para mejorar la producción agrícola. En el año 2002, los países miembros de la OECD aplicaron en total 929 mil toneladas de plaguicidas (OECD, 2002). Se estima que el uso anual promedio de plaguicidas en esos países es de 0.22 ton $/ \mathrm{km}^{2}$. En México, en el período comprendido del año 2004 al 2009, se aplicaron 123 mil toneladas de plaguicidas, lo que representa el $16 \%$ del total a nivel mundial (FAOSTAT, 2011). Actualmente, de los 90 plaguicidas que han sido restringidos en los Estados Unidos, 30 se siguen usando en México (organoclorados y organofosfo- rados) (Tafoya, 2008), lo cual representa una amenaza potencial para la salud.

La atrazina (6-chloro- $\mathrm{N}^{2}$-ethyl- $\mathrm{N}^{4}$-isopropyl-1,3,5triazine-2,4-diamine) con número de CAS 191224-9, es un herbicida selectivo sistémico, utilizado como control -pre y post- emergente de malezas de hoja ancha y pastos en general, que actúa por vía radical y se acumula en las hojas impidiendo el proceso fotosintético de las plantas (González-Márquez y Hansen, 2009). Es el herbicida más utilizado en el cultivo de maíz (Zea mays) alrededor del mundo, principalmente en países como Estados Unidos, China, Brasil, México y Argentina (LeBaron et al., 2008; Kim et al., 2011). Su uso tan extendido se debe a que mejora de forma importante los rendimientos agrícolas al eliminar la competencia del cultivo con especies no deseadas. Por otra parte, su uso constituye un factor de disrupción en los ciclos biogeoquímicos, ya que la adición de compuestos orgánicos exógenos modifica la estructura funcional de la comunidad microbiana (El-Bestawy et al., 2013) y por consiguiente la regulación biológica de las funciones en los suelos (Kibblewhite et al., 2008). Se ha reportado además, que el uso de la atrazina implica el riesgo de alterar la dinámica del carbono $(\mathrm{C})$ y nitrógeno (N) en el suelo (Miller y Doxtader, 1995; Laursen y Carlton, 1999), así como el riesgo de contaminación ambiental y su consecuente afectación a la salud humana. La atrazina ha sido detectada en el agua superficial con concentraciones entre 4.6 y $15.0 \mu \mathrm{g} / \mathrm{L}$ (DR 063 en Sinaloa, México) y en el agua subterránea con valores entre 0.1 y $0.4 \mu \mathrm{g} / \mathrm{L}$ (Valle de San Joaquín en California, USA) (Domagalski y Dubrovsky, 1992; Hernández-Antonio y Hansen, 2011; Lerch et al., 2011), las cuales se encuentran por encima de la legislación internacional cuyo límite en agua para consumo humano en los Estados Unidos de América es de $3 \mu \mathrm{g} / \mathrm{L}$ (USEPA, 2003) y 0.1 g $/ \mathrm{L}$ en la Comunidad Europea (European Parliament, 1998). Los efectos tóxicos de la atrazina en la salud humana pueden ser agudos como irritación severa de ojos y piel o crónicos como los relacionados con daños reproductivos. Por esta razón, se prohibió su uso en la 
Comunidad Europea desde el año 2004.

Los suelos de manera natural son amortiguadores de contaminantes, ya que al pasar a través de la zona vadosa se puede prevenir y/o retrasar su llegada al acuífero. Al infiltrarse el agua a través de los poros del suelo, esta moviliza a los contaminantes en forma disuelta o adsorbida, bajo sistemas coloidales o partículas dispersas en la fase acuosa que favorecen el contacto con la matriz del suelo donde ocurren procesos físicos, químicos y biológicos que definen el destino ambiental de los contaminantes. En el caso de la atrazina, al ser una base débil (pKa de 1.7), se encuentra en forma básica como molécula y sin carga formal en suelos con valores de $\mathrm{pH}>1.7$, y en esta condición no es retenida electrostáticamente sobre las superficies con carga neta negativa de algunos componentes del suelo (Dragun, 1998). La adsorción es un proceso clave para que un compuesto esté disponible para ser degradado por los microorganismos o bien, transportado hacia estratos más profundos. Se sabe que la adsorción de atrazina ocurre principalmente en la fracción orgánica del suelo (Nicholls, 1988; Shea, 1989; Barriuso et al., 1992; Payá-Pérez et al., 1992; Dousset et al., 1994). Al respecto, se ha reportado que existe una gran afinidad de la atrazina por los ácidos húmicos (Dutta et al., 2015), lo cual se incrementa en función del grado de aromaticidad de las moléculas orgánicas (Kulikova y Perminova, 2002). Por otro lado, Loiseau y Barriuso (2002) determinaron que en la fracción mineral fina del suelo (partículas de tamaño inferior a $20 \mu \mathrm{m}$ ), es donde se absorbe la mayor proporción de atrazina. Una vez que la atrazina es retenida en la zona vadosa, puede ser metabolizada por los microorganismos del suelo (Ross et al., 2006). Esta capacidad de degradación microbiana está considerada como un atributo de la capacidad buffer de los suelos ante sustancias contaminantes (Blum y Eswaran, 2004).

Existen evidencias que indican que el uso continuo de atrazina, genera resistencia en las malezas (Shukla y Devine, 2008), lo que resulta en el detrimento del objetivo para el cual es aplicada. Para eludir la resistencia de las malezas al efec- to del herbicida, las estrategias utilizadas más comúnmente son: el incremento de la dosis aplicada periódicamente o bien, el uso de mezclas de compuestos químicos entre los cuales existe sinergia, es decir, el efecto destructor de ambos es superior a la suma del efecto individual de cada uno, lo cual supera la capacidad de amortiguamiento del suelo, y genera un detrimento en la estabilidad del mismo (Lal, 1993), esta última entendida como la susceptibilidad de cambio en sus propiedades bajo alguna perturbación, lo cual es un rasgo común de los suelos en el Antropoceno. En esta era, la actividad del hombre está modificando las condiciones ambientales a nivel regional, lo que provoca impactos sobre la biodiversidad, el clima y la salud humana (Pimm et al., 1995; Myers y Patz, 2009; Huber y Knutti, 2012). De este modo, estudios detallados sobre el efecto que las actividades humanas, como el mal manejo del suelo y el uso de sustancias orgánicas exógenas como el herbicida atrazina, tienen sobre el recurso, son necesarios.

Entender de qué manera las prácticas agrícolas afectan a la función amortiguadora del suelo es indispensable para la preservación del recurso. En este contexto, el presente trabajo hace un recorrido por diversas localidades del país que utilizan al herbicida atrazina para el control de malezas de hoja ancha en la producción de maíz, con el objetivo de evaluar uno de los principales procesos que definen el destino ambiental del herbicida en el suelo, la adsorción y desorción en el horizonte superficial de tres diferentes tipos de suelos: Andosol, Feozem y Vertisol bajo diferente manejo agrícola (agricultura convencional, labranza cero y agricultura de temporal). En cada uno de ellos se valora el efecto que las prácticas agrícolas locales han tenido en las propiedades del suelo y por ende, en la movilidad del herbicida. El objetivo del presente trabajo fue evaluar la capacidad de adsorción y desorción de atrazina en el horizonte superficial de cada suelo, a partir del coeficiente de distribución suelo/solución $\left(\mathrm{K}_{\mathrm{d}}\right)$ obtenido en experimentos batch. El resultado obtenido se relacionó con las características de los suelos que tienen que ver con la retención del herbicida, notablemente 
el contenido de MO y arcillas, y se evaluó el efecto que las prácticas agrícolas locales han podido tener en ellos. Este manuscrito se trata de un trabajo de recapitulación de artículos ya publicados (suelos de la cuenca de Valle de Bravo y del Estado de México) junto con nuevas investigaciones realizadas recientemente en el grupo de investigación de los autores (en suelos de Hidalgo regados con agua residual).

\section{Materiales y Métodos}

\subsection{SITIOS Y SUELOS ESTUDIADOS}

Los sitios seleccionados se localizan en la zona centro de México (Figura 1). La porción sur de esta región se caracteriza por ser una zona montañosa con alturas mayores de $2000 \mathrm{msnm}$, cubierta en su mayoría por rocas volcánicas cenozoicas y clima subhúmedo con lluvias en verano. La porción norte por su parte, se caracteriza por tener una morfología de grandes cuencas continentales rellenas de sedimentos aluviales y lacustres y clima semiárido (Nieto-Samaniego et al., 2005).

Se seleccionaron tres diferentes tipos de suelos: Andosol, Feozem y Vertisol. Los Andosoles cubren el $1.2 \%$ del territorio mexicano (INEGI, 1999) y representan un recurso importante para la agricultura en varias regiones del mundo, lo cual se debe a la presencia de materiales amorfos que les dan características distintivas como: la baja densidad aparente, alto contenido de $\mathrm{MO}$, alta porosidad y muy alta capacidad de retención de humedad, todas ellas favorables para el desarrollo de raíces y crecimiento de las plantas (Nanzyo, 2002). Los Feozems y Vertisoles son suelos medianamente profundos y ricos en $\mathrm{MO}$; en conjunto representan el $18.0 \%$ de los suelos del país (SEMARNAT-CP-INEGI, 1999). En la región centro de México, los Feozems se localizan en planicies lacustres y fluvioaluviales o sobre depósitos volcanoclásticos y aluviocoluviales en zonas templadas y húmedas, mientras que los Vertisoles se localizan en planicies aluviales y piedemontes, formados a partir de depósitos de origen volcanoclástico y material aluvial en zonas subhúmedas y secas ( $\mathrm{Ga}-$ rrido-Pérez et al., 2006). Los Feozems tienen alto contenido de $\mathrm{MO}$ y textura media a fina, drenaje interno moderado y buena aireación. Por su parte, los Vertisoles tienen una textura muy fina que se caracteriza por un drenaje interno y aireación pobre, y poseen alto contenido de arcilla. El conjunto de características de estos suelos, hacen que los Feozems y Vertisoles hacen que estos tipos de suelos sean empleados para la agricultura en diferentes formas de manejo, el $35 \%$ son destinados para la agricultura de temporal y el $45 \%$ para la agricultura de riego.

El Andosol estudiado se localiza dentro de la cuenca hidrológica Valle de Bravo-Amanalco, la cual aporta el $40 \%$ del agua potable consumida en el área metropolitana de la Ciudad de México y Toluca (CGVM, 2016). El uso de suelo originalmente era forestal con bosque de pino-encino y esta vegetación ha sufrido una fragmentación a usos de agricultura de temporal y pastizales. El suelo dominante fue clasificado como Andosol Pachic (FAO-ISRIC e ISSS, 1998). En el sitio se siembra maíz de temporal y la producción es apenas suficiente para satisfacer la demanda de los habitantes de la zona.

El Feozem se muestreó en dos parcelas agrícolas que pertenecen a la estación experimental "El Batán", del Centro Internacional de Mejoramiento de Maíz y Trigo. El tipo de suelo se clasificó como un Feozem háplico arcilloso (IUSS, Working Group WRB, 2006). Se seleccionaron dos parcelas bajo diferentes sistemas de manejo agrícola: (1) labranza cero con rotación de cultivos maíz/ trigo y retención de residuos (LZ) y (2) labranza convencional con monocultivo de maíz (LC), sin retención de residuos.

En el caso del Vertisol, se muestrearon tres sitios localizados a $80 \mathrm{~km}$ de la Ciudad de México, en la región conocida como el Valle del Mezquital en el estado de Hidalgo, región que recibe desde hace 100 años, las aguas residuales de la Ciudad de México para ser utilizadas en el riego de campos agrícolas. En este sitio se muestrearon tres sue- 


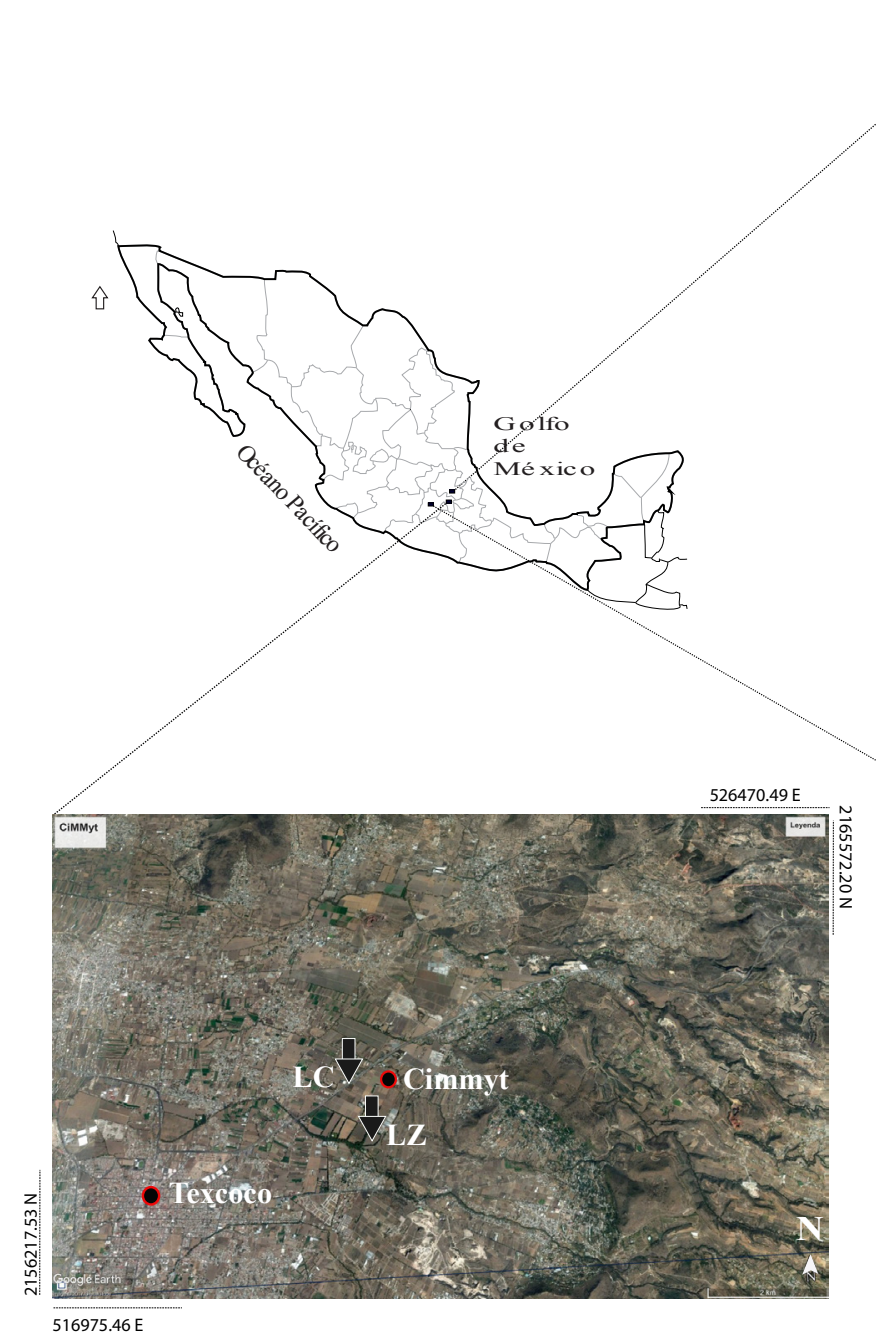

El Batán, Texcoco, Estado de México

$\checkmark$ Sitios en donde se tomaron las muestras en suelo tipo Feozem en diferentes parcelas experimentales del CIMMYT en sistemas de labranza convencional (LC) y labranza cero (LZ) .

Figura 1 Localización de los sitios estudiados.

los agrícolas clasificados como Vertisoles éutricos (FAO-ISRIC e ISSS, 1998). El primero de ellos se recolectó en una parcela de temporal ubicada dentro de la localidad Tetepango (VT), los otros dos sitios, ubicados en las localidades de Ulapa-Tetepango y Ulapa, que han sido regados con agua residual durante un periodo de 56 (V56) y 100 (V100) años, respectivamente.

Los tres tipos de suelo estudiados se encuentran bajo diferentes escenarios de manejo agrícola, teniendo en común el uso de atrazina para el control

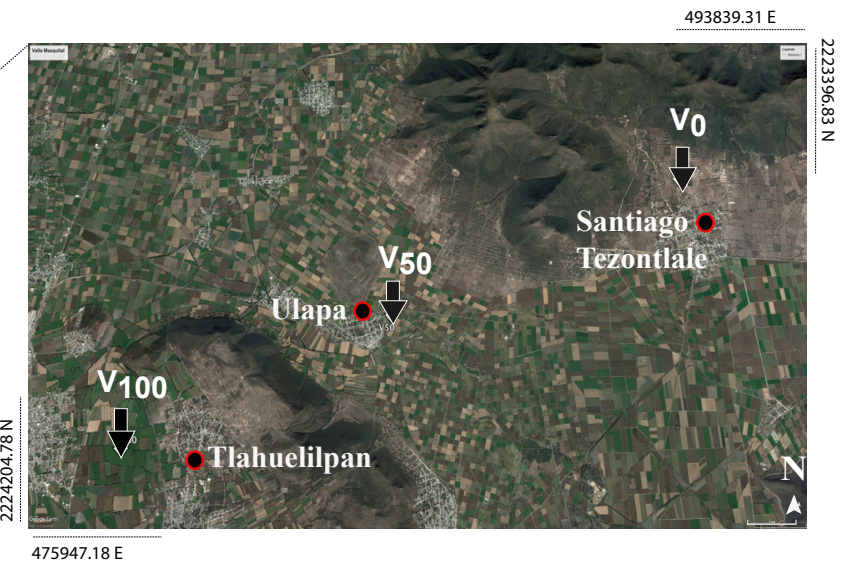

\section{Valle del Mezquital-Hidalgo}

I Sitios en donde se tomaron las muestras de suelo que representan las zonas con suelos Vertisoles regados con agua residual por 100 años $\left(\mathrm{V}_{100}\right)$, 50 años $\left(\mathrm{V}_{50}\right)$ y de temporal $\left(\mathrm{V}_{0}\right)$.

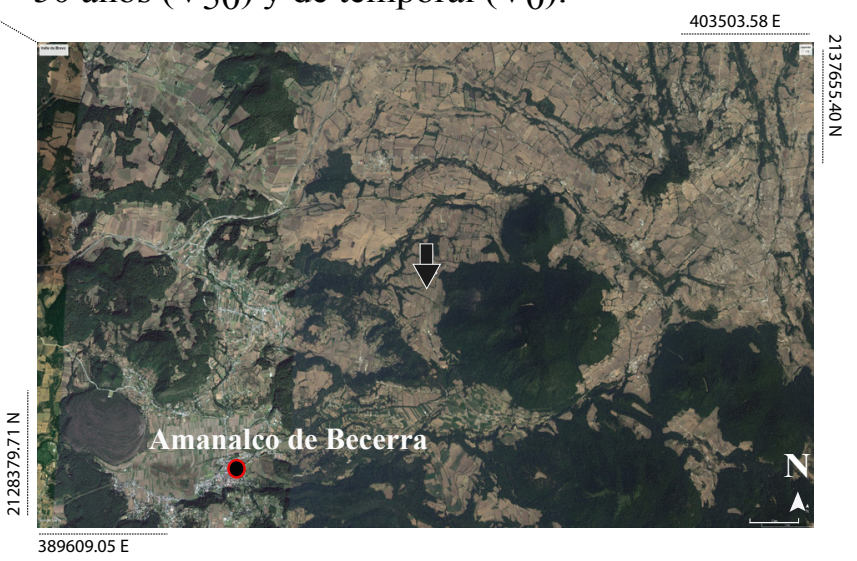

\section{Valle de Bravo- Estado de México}

$\downarrow$ Sitio en donde se tomó la muestra de suelo que representa el Andosol en laderas con cambio de uso de suelo, de uso forestal a agricultura de temporal.

de malezas de hoja ancha en los cultivos de maíz. En la Tabla 1 se presenta la localización de los sitios estudiados y las prácticas de manejo agrícola en cada uno de ellos, y en la Tabla 2 se muestran las principales características fisicoquímicas de los suelos.

\subsection{EL HERBICIDA}

La atrazina es un polvo blanco con un valor de pKa de $1.7\left(20^{\circ} \mathrm{C}\right)$, que presenta una presión 
Tabla 1. Sitios estudiados, localización y prácticas agrícolas.

\begin{tabular}{|c|c|c|c|c|c|}
\hline Localidad & Sitio & Tipo de suelo & Tipo de agricultura & $\begin{array}{c}\text { Dosis de atrazina } \\
\text { aplicada en campo } \\
(\mathrm{kg} / \mathrm{ha})^{\mathrm{a}}\end{array}$ & $\begin{array}{c}\text { Tiempo de aplicación } \\
\text { (años) }\end{array}$ \\
\hline $\begin{array}{c}\text { Valle de Bravo, Estado } \\
\text { de México }\end{array}$ & La Loma & Andosol & $\begin{array}{c}\text { Temporal, } \\
\text { laboreo manual, } \\
\text { consumo familiar }\end{array}$ & 1 & - \\
\hline \multirow{2}{*}{$\begin{array}{l}\text { Texcoco, Estado de } \\
\text { México }\end{array}$} & El Batán & Feozem & $\begin{array}{l}\text { Sitio experimental, } \\
\text { riego por aspersión }\end{array}$ & 1.85 & - \\
\hline & Tetepango & Vertisol & Temporal & 1.12 & 15 \\
\hline \multirow{2}{*}{$\begin{array}{c}\text { Valle del Mezquital, } \\
\text { Hidalgo }\end{array}$} & Ulapa-Tetepango & Vertisol & $\begin{array}{l}\text { Uso permanente, } \\
\text { riego por inundación, } \\
\text { agua residual (56 años) }\end{array}$ & 1.12 & 15 \\
\hline & Ulapa & Vertisol & $\begin{array}{l}\text { Uso permanente, } \\
\text { riego por inundación, } \\
\text { agua residual (100 años) }\end{array}$ & 1.12 & 15 \\
\hline
\end{tabular}

${ }^{a}$ Ingrediente activo

Tabla 2. Propiedades fisicoquímicas de los suelos evaluados.

\begin{tabular}{|c|c|c|c|c|c|c|c|c|c|c|}
\hline \multirow{2}{*}{ Suelo } & \multicolumn{3}{|c|}{ Textura } & \multirow{2}{*}{ Tipo de arcilla } & \multirow{2}{*}{$\begin{array}{l}\text { COD } \\
(\mathrm{g} / \mathrm{kg})\end{array}$} & \multirow{2}{*}{ pH } & COS & $\mathbf{A F}$ & $\mathbf{A H}$ & $\mathbf{H}$ \\
\hline & $\operatorname{arena}(\%)$ & $\operatorname{limo}(\%)$ & arcilla (\%) & & & & \multicolumn{4}{|c|}{$(\mathrm{g} / \mathrm{kg})$} \\
\hline Andosol & 23 & 66 & 11 & $\begin{array}{c}\text { alofano }^{(a)} \\
\text { ferrihidrita }^{(b)}\end{array}$ & 10.4 & 6.5 & 37.3 & 7.1 & 14.9 & 4.9 \\
\hline Feozem $_{(\mathrm{LZ})}$ & 25 & 37 & 38 & - & 0.51 & 6 & 28.5 & 1.8 & 6.4 & 18.1 \\
\hline Feozem $_{(\mathrm{LC})}$ & 25 & 37 & 38 & - & 0.45 & 6.1 & 14.8 & 1.1 & 6.4 & 6.8 \\
\hline Vertisol $_{\text {(VT) }}$ & 18 & 28 & 54 & montmorillonita & - & 7 & 30.6 & - & - & - \\
\hline Vertisol $_{(\mathrm{V} 56)}$ & 19 & 49 & 32 & montmorillonita & - & 7.2 & 16.8 & - & - & - \\
\hline Vertisol $_{(\mathrm{V} 100)}$ & 10 & 41 & 49 & montmorillonita & - & 7.3 & 26.6 & - & - & - \\
\hline
\end{tabular}

(LZ) labranza cero; (LC) labranza convencional; (VT) Vertisol temporal; (V56) Vertisol 56 años bajo riego con agua residual;

(V100) Vertisol 100 años bajo riego con agua residual

COD (carbono orgánico disuelto)

COS (materia orgánica del suelo)

AF (ácidos fúlvicos), AH (ácidos húmicos), H (huminas)

(a)Contenido de alofano $18.5 \%$, (b) ferrihidrita $3.2 \%$ (Parfitt, 1990).

Nota: El pH del suelo se midió en agua utilizando una relación suelo:agua de 1:2.5. El carbono orgánico total (TOC) se analizó mediante combustión en seco utilizando un analizador automático de carbono (TOC, Shimadzu 5000). La textura del suelo se determinó mediante el método de la pipeta después de eliminar la materia orgánica con peróxido y la dispersión del suelo con hexametafosfato de sodio. Los ácidos húmicos (AH), ácidos fúlvicos (AF) y las huminas (H) del suelo, así como el carbono orgánico disuelto (COD) se aislaron de las muestras de suelo de acuerdo con los procedimientos convencionales basados en la extracción con pirofosfato hidróxido-sodio, precipitación con $\mathrm{HCl}$ a $\mathrm{pH} \sim 2$, purificación suave mediante disolución sucesiva de $\mathrm{NaOH}$ y etapas de precipitación con $\mathrm{HCl}$ (Stevenson, 1994). 
de vapor de $2.9 \times 10^{-7} \mathrm{~mm} \mathrm{Hg}$ a $298 \mathrm{~K}$ y una solubilidad en agua de $33 \mathrm{mg} / \mathrm{L}\left(20^{\circ} \mathrm{C}\right)$ a $\mathrm{pH}$ de 7 (Tomlin, 1997) y un coeficiente de partición octanol/agua (log Kow) de 2.6 (Cejudo-Espinosa et al., 2009).

\subsection{ANÁLISIS DE LA GAPACIDAD DE ADSORGIÓN - DESORGIÓN DE LOS SUELOS ESTUDIADOS}

Se realizaron experimentos utilizando muestras colectadas de suelo superficial en cada uno de los sitios estudiados, las muestras fueron tamizadas frescas $(<2 \mathrm{~mm})$ y almacenadas a $4^{\circ} \mathrm{C}$ hasta ser utilizadas en los experimentos correspondientes.

Se llevaron a cabo los experimentos estándar en lote para obtener las isotermas de adsorción de atrazina en los diferentes tipos de suelo, así como una etapa de desorción. En todos los suelos, las isotermas se determinaron en cinco concentraciones iniciales de atrazina, las cuales se establecieron dentro de un intervalo en particular (Tabla 3) con base en la dosis aplicada en cada sitio (Tabla 1). Las soluciones de atrazina utilizadas fueron preparadas mediante la dilución de una solución stock de atrazina disuelta en metanol (ChemService, West Chester, USA, $99.8 \%$ pureza), de acuerdo con Montaño (2013). Para verificar la posible retención del analito estudiado en el material de los contenedores utilizados, se prepararon disoluciones control para todos los niveles de concentración de atrazina empleados en los experimentos de sor- ción, las cuales fueron sometidas al protocolo completo utilizado para la obtención de las isotermas. Finalmente, dichas disoluciones fueron analizadas mediante la técnica de Cromatografía de Líquidos de Alta Resolución (HPLC) y un detector UVDAD, encontrándose la ausencia de cambios en los valores de la concentración inicial después del tratamiento. Además, para descartar el efecto de la luz como factor promotor del proceso de fotodegradación, durante la realización de las diferentes etapas del experimento, las muestras se colocaron en charolas cubiertas con papel aluminio.

La relación suelo-solución fue de 1:2.5 en todos los suelos, excepto en el Vertisol de la región del Valle del Mezquital, en donde se usó una relación 1:5. El tiempo de equilibrio $(24 \mathrm{~h})$ se determinó con base en los experimentos cinéticos preliminares de acuerdo con Roy et al. (1991). Las alícuotas de suelo, expresadas en masa seca, se mezclaron con el volumen requerido de la solución utilizada como electrolito (Tabla 3). Todas las muestras de los diferentes tipos de suelos se equilibraron mediante agitación en un agitador orbital por un periodo de tiempo de equilibrio de $24 \mathrm{~h}$ a $\mathrm{pH}$ del suelo. Posteriormente, se les agregó el estándar de atrazina y se agitaron nuevamente. Luego de 24 $\mathrm{h}$, las suspensiones se centrifugaron a $2500 \mathrm{rpm}$ por 10 min y finalmente se filtró una alícuota de 1 $\mathrm{mL}(13 \mathrm{~mm}$ x $0.45 \mu \mathrm{m}$ - nylon Millex-NH), la cual se transfirió a viales de vidrio de color ámbar de $1 \mathrm{~mL}$ para su posterior análisis mediante HPLC-

Tabla 3. Condiciones experimentales de las isotermas de adsorción - desorción de atrazina.

\begin{tabular}{|c|c|c|c|c|c|c|}
\hline Suelo & $\begin{array}{c}\text { Intervalo de } \\
\text { concentración } \\
(\mu \mathrm{g} / \mathrm{g})\end{array}$ & $\begin{array}{c}\text { Relación } \\
\text { suelo:solución }\end{array}$ & $\begin{array}{l}\text { Electrolito } \\
(10 \mathrm{mM})\end{array}$ & $\begin{array}{c}\text { Velocidad } \\
\text { agitación } \\
\text { /temperatura }\end{array}$ & $\begin{array}{l}\text { Velocidad } \\
\text { centrifugado } \\
\text { /tiempo }\end{array}$ & $\begin{array}{l}\text { Método analítico } \\
\text { (cuantificación } \\
\text { atrazina) }\end{array}$ \\
\hline Andosol & $1.7-13.6$ & 01:02.5 & $\begin{array}{c}\text { Solución } \\
\text { reconstituida }^{(a)}\end{array}$ & $40 / 20$ & $1050^{(b)} / 30$ & HPLC/detector UV \\
\hline Feozem & $0-95$ & 01:02.5 & $\mathrm{CaCl}_{2}$ & $40 / 20$ & $1050^{(b)} / 30$ & $\begin{array}{c}\text { HPLC/detector } \\
\text { DAD }\end{array}$ \\
\hline Vertisol & ago-40 & 01:05 & $\mathrm{CaCl}_{2}$ & $120 / 20$ & $2500^{(\mathrm{c})} / 10$ & $\begin{array}{c}\text { HPLC/detector UV- } \\
\text { DAD }\end{array}$ \\
\hline
\end{tabular}

(a) $0.3 \mathrm{mM} \mathrm{KCl}, 1 \mathrm{mM}$ de $\mathrm{CaCl}_{2}, 0.4 \mathrm{mM} \mathrm{MgCl}$ y $0.4 \mathrm{mM} \mathrm{NaCl}$

Velocidad de agitación (rpm); temperatura ( ${ }^{\circ} \mathrm{C}$ )

Velocidad de centrifugado: (b)gravedades; ${ }^{(c)} \mathrm{rpm}$; tiempo (min)

HPLC (high pressure liquid chromatography); UV (ultra violeta); DAD (detector de arreglo de diodos)

Nota: los experimentos se realizaron al pH de los suelos, ver Tabla 2. 
UV-DAD. En la Tabla 3 se muestran las condiciones experimentales para cada tipo de suelo.

Las concentraciones de atrazina adsorbida S $(\mu \mathrm{g} / \mathrm{g})$ se determinaron de acuerdo con la Ecuación 1:

$$
S=\frac{\left(C_{0} V_{0}-C V\right)}{m} \quad \text { (Ecuación 1) }
$$

Donde, $C_{0}$ es la concentración inicial de atrazina $(\mu \mathrm{g} / \mathrm{mL}), \mathrm{C}$ es la concentración de equilibrio medida en el sobrenadante después de la agitación $(\mu \mathrm{g} / \mathrm{mL}), V$ y $V_{0}$ corresponden al volumen en equilibrio y al volumen inicial de la solución de atrazina $(\mathrm{mL})$, respectivamente, y $m$ es el peso seco del suelo $(\mathrm{g})$.

Para el análisis de la desorción del herbicida desde el suelo, después de la etapa de centrifugado, se removió toda la fase acuosa. El volumen de solución removido, fue sustituido por el mismo volumen de la solución del electrolito. El volumen preciso, removido o agregado, de la solución del electrolito, se registró para cada muestra. Posteriormente, las muestras se agitaron nuevamente por $24 \mathrm{~h}$ y después se centrifugaron bajo las mismas condiciones que en la etapa de adsorción. El sobrenadante se analizó para determinar la concentración de atrazina.

La concentración de atrazina desorbida $\left(S_{\text {des }}\right)$ en la etapa de desorción $(\mu \mathrm{g} / \mathrm{mL})$, fue estimada de acuerdo con la Ecuación 2:

$$
S_{\text {des }}=\frac{\left(C V_{\text {en_suelo }}+S m\right)-C_{1} V_{1}}{m} \text { (Ecuación 2) }
$$

Donde, $V_{\text {en_sulo }}$ y $V_{l}$ son los volúmenes de la solución del suelo restante de la etapa de adsorción y el volumen del sobrenadante de la etapa de desorción $(\mathrm{mL})$, respectivamente, y $C_{1}$ es la concentración de equilibrio medida en el sobrenadante después de la etapa de desorción $(\mu \mathrm{g} / \mathrm{mL})$.

Para entender el efecto y la importancia del contenido de MO del suelo sobre la adsorción de atrazina, así como la participación de las arcillas en este proceso, el procedimiento de adsorción se realizó utilizando también al Andosol y al Vertisol sin contenido de MO.
En el caso del Andosol, la eliminación de la MO se realizó a partir del método de oxidación húmeda, utilizando peróxido de hidrógeno $\left(\mathrm{H}_{2} \mathrm{O}_{2}\right)$ como agente oxidante. Para ello, se tomaron tres sub-muestras de $50 \mathrm{~g}$ cada una, tamizadas previamente $(<2 \mathrm{~mm})$ y secadas a temperatura ambiente. Después cada sub-muestra se mezcló con 100 $\mathrm{mL}$ de $\mathrm{H}_{2} \mathrm{O}_{2}$ al $30 \%$. Esta suspensión se colocó en un sistema abierto a $60{ }^{\circ} \mathrm{C}$ en un baño de arena por varios días, hasta que se observó un cambio de color en la suspensión. Una vez que esto ocurrió, el suelo tratado se lavó con agua destilada.

En el caso del Vertisol, el tratamiento utilizado para eliminar la MO fue mediante el uso de hipoclorito de sodio $(\mathrm{NaClO})$ como agente oxidante (Siregar et al., 2005). Se tomaron tres sub-muestras de $100 \mathrm{~g}$ (secadas previamente a temperatura ambiente, y tamizadas a $<2 \mathrm{~mm}$ ). Después, cada sub-muestra se mezcló con $1 \mathrm{~L}$ de $\mathrm{NaClO}$ al $6 \%$ ajustado a un $\mathrm{pH}=8$ con ácido clorhídrico concentrado, y se colocaron en un agitador orbital a $120 \mathrm{rpm}$ por un periodo de $24 \mathrm{~h}$. Transcurrido ese tiempo, las muestras se centrifugaron durante 10 minutos a 2500 rpm y se decantó el sobrenadante; todo este proceso se repitió en dos ocasiones más. El suelo tratado con $\mathrm{NaClO}$ se lavó con 1 L de una solución de cloruro de sodio $1 \mathrm{M}$, agitándolo a $120 \mathrm{rpm}$ por 24 h, y después centrifugándolo por 10 minutos a $2500 \mathrm{rpm}$. Una vez terminado este proceso, el suelo tratado se transfirió a membranas semipermeables (Medicell International, peso molecular 12000 - 14000 Da), para ser dializado colocando las membranas en un contenedor lleno con agua destilada. Durante el proceso de diálisis, el agua destilada utilizada para tal fin se renovó y monitoreó constantemente hasta que la conductividad eléctrica fue $<40 \mu \mathrm{S} / \mathrm{cm}$, momento en el cual se consideró que la muestra estaba libre de cloruros. Al final de todo el proceso las sub-muestras se liofilizaron.

\subsection{GUANTIFIGACIÓN DE ATRAZINA}

El análisis de atrazina en las muestras del Vertisol se llevó a cabo mediante HPLC, utilizando un equipo constituido de 2 bombas binarias (modelo 
1525), un auto-muestreador (modelo 717 plus) y un detector UV-DAD a una $\lambda=220 \mathrm{~nm}$ (modelo 2998) todos ellos de la marca Waters ${ }^{\circledR}$. Como fase estacionaria se utilizó una columna de fase reversa (Spherisorb ODS2 de 250 x $4 \mathrm{~mm}$, empacada con partículas de tamaño de $5 \mu \mathrm{m}$, Waters $\left.{ }^{\circledR}\right)$, y como fase móvil una mezcla de un buffer de fosfatos de $\mathrm{pH}=7.2$-acetonitrilo en una relación $60: 40 \mathrm{v} / \mathrm{v}$ en modo isocrático, a un flujo de $1 \mathrm{~mL} / \mathrm{min}$, de acuerdo con lo reportado por Cortes (2016). Para las muestras del Andosol, la cuantificación de atrazina se llevó a cabo de acuerdo con lo reportado en Prado et al. (2014a), y en el caso de las muestras del Feozem la metodología se describe en Prado et al. (2014b).

\section{Resultados y discusión}

El destino de la atrazina en el suelo depende en gran medida de los procesos de adsorción y desorción, que definen la lixiviación del herbicida, lo cual implica que deben de ser tomados en cuenta al predecir el transporte del mismo en el suelo (Jarvis et al., 1991; Simunek et al., 1999), así también, la adsorción controla la persistencia y retención irreversible de pesticidas en el suelo (de Jonge et al., 1996). A continuación se presentan los resultados obtenidos en cada sitio estudiado, así como el análisis y la discusión de los mismos.

\subsection{ADSORGIÓN - DESORGIÓN DE ATRAZINA DE UN ANDOSOL BAJO AGRIGULTURA DE TEMPORAL}

La Figura 2 muestra las isotermas de adsorción y desorción del Andosol con MO (Figura 2a) y sin MO (Figura 2b), en todos los casos se observa una relación lineal entre la cantidad de atrazina adsorbida y la concentración en equilibrio. El coeficiente de adsorción fue mayor en el suelo con MO que en suelo sin MO (Tabla 4), resultado que nos permite decir que la MO del suelo es el principal adsorbente, y en menor proporción la fracción mineral, como el alofano y la ferrihidrita identificados en este suelo (Prado et al., 2007). La capacidad de adsorción de atrazina por minerales amorfos como el alofano ya ha sido documentada por otros autores. Close et al. (2003) reportaron que al aumentar el alofano aumenta el $\mathrm{K}_{\mathrm{d}}$, en suelos con contenidos del mineral entre 5 y $12 \%$. Así mismo, Baskaran et al. (1996) observaron que la adsorción de atrazina en suelos alofánicos se incrementa después de remover el carbono del suelo.

Por su parte, Ben-Hur et al. (2003) mencionan que un indicador de la participación de la fracción mineral en la adsorción de atrazina, es la relación

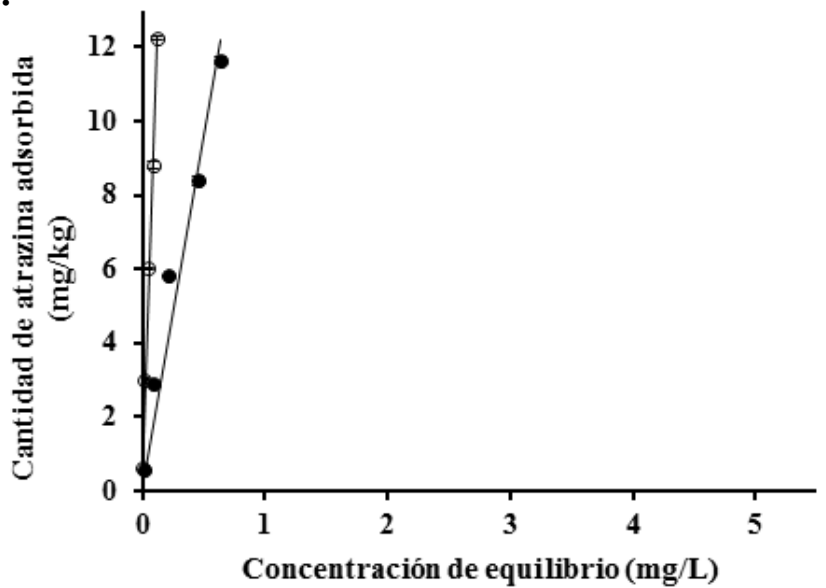

b.

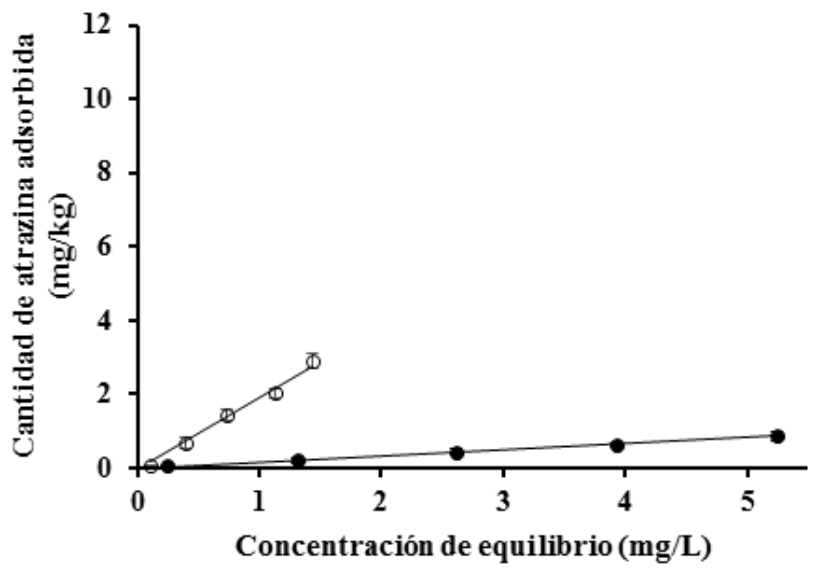

Figura 2 Isotermas de adsorción de atrazina (símbolos rellenos) y desorción (símbolos huecos), se muestra en $a$. Suelo Andosol de la subcuenca La Loma, Valle de Bravo. En b. Suelo Andosol bajo tratamiento oxidativo con $\mathrm{H}_{2} \mathrm{O}_{2}$. Se indica la línea de ajuste lineal y las barras de desviación en ambos casos. 
Tabla 4. Coeficientes de distribución $\left(\mathrm{K}_{\mathrm{d}}\right)$ de adsorción y desorción de atrazina.

\begin{tabular}{|c|c|c|c|c|c|c|}
\hline \multirow{2}{*}{ Suelo } & \multicolumn{2}{|c|}{$\begin{array}{l}\text { Suelo con MOS } \\
\qquad K_{d}(\mathrm{~L} / \mathrm{kg})\end{array}$} & \multirow{2}{*}{$\mathbf{K}_{\mathrm{oc}}$} & \multirow{2}{*}{$\begin{array}{c}\text { Relación } \\
\text { Arcilla/CO }\end{array}$} & \multicolumn{2}{|c|}{$\begin{array}{c}\text { Suelo sin MOS } \\
\mathbf{K}_{\mathbf{d}}(\mathrm{L} / \mathrm{kg}) \\
\end{array}$} \\
\hline & Adsorción $\left(\mathbf{R}^{2}\right)$ & Desorción $\left(\mathbf{R}^{2}\right)$ & & & Adsorción $\left(\mathbf{R}^{2}\right)$ & Desorción $\left(\mathbf{R}^{2}\right)$ \\
\hline Andosol & $19.3(0.93)$ & $101.9(0.92)$ & 520 & 3 & $1.6(0.99)$ & $1.9(0.98)$ \\
\hline Feozem $_{(\mathrm{LZ})}$ & $1.1(0.95)$ & $79.4(0.83)$ & 56 & 28 & NR & NR \\
\hline Feozem $_{(\mathrm{LC})}$ & $0.92(0.96)$ & $89.4(0.78)$ & 76 & 29 & NR & NR \\
\hline Vertisol $_{(\mathrm{VT})}$ & $3.80(0.72)$ & $25.25(0.86)$ & 124 & 18 & $2.05(0.80)$ & $4.04(0.74)$ \\
\hline Vertisol $_{(\mathrm{V} 56)}$ & $11.08(0.79)$ & $159.43(0.94)$ & 660 & 19 & $1.96(0.86)$ & $7.16(0.93)$ \\
\hline Vertisol $_{(\mathrm{V} 100)}$ & $9.02(0.75)$ & $42.36(0.95)$ & 339 & 18 & $2.09(0.98)$ & $5.03(0.81)$ \\
\hline
\end{tabular}

(LZ) labranza cero; (LC) labranza convencional; (AI) agrícola intensivo; (VT) Vertisol temporal;

(V56) Vertisol 56 años bajo riego con agua residual; (V100) Vertisol 100 años bajo riego con agua residual

$\mathbf{R}^{2}$ (coeficiente de correlación ajustado)

$\mathrm{K}_{\mathrm{oc}}$ (coeficiente de adsorción normalizado al contenido de $\mathrm{CO}$ )

Arcilla $(\mathrm{g} / \mathrm{kg})$

CO (carbono orgánico, g/kg)

MOS (materia orgánica del suelo)

NR (no realizado)

arcilla/carbono orgánico $(\mathrm{CO})$ del suelo (arcilla/ $\mathrm{CO})$. Para los autores un contenido de arcilla superior a 30 veces el contenido de $\mathrm{CO}$ del suelo, indica que la fracción mineral es la principal responsable de la retención del herbicida. En el caso del Andosol estudiado, el contenido de arcilla es apenas 3 veces el contenido de CO (Tabla 4). El resultado además confirma lo citado por Close et al. (2003), quienes indican que normalmente se considera que la adsorción de pesticidas está controlada principalmente por la MO del suelo.

La capacidad de adsorción de atrazina del suelo disminuye en un $99 \%$ al eliminar la MO. El rol de la fracción orgánica del suelo como el principal adsorbente de atrazina ha sido documentado por varios autores (Stevenson, 1994; Baskaran et al., 1996). La calidad de la fracción orgánica del suelo también define una mayor o menor afinidad del suelo por el herbicida. Existen trabajos en los cuales se ha reportado que para ciertos herbicidas, las características de la fracción orgánica del suelo son de gran importancia en su retención, pero esta afinidad no es tan fuerte para otros herbicidas (Bailey y White, 1964; Hayes, 1970; Arienzo y Buondonno, 1993; Riise et al., 1994; Jenks et al.,
1998; Bekbolet et al., 1999). Al respecto, Mudhoo y Garg (2011) señalan que la atrazina tiene mayor afinidad por los compuestos aromáticos e hidrófobos, los cuales pueden ser residuos orgánicos con diferente origen (Kögel-Knaber, 2002) o sustancias derivadas de resíntesis microbiana, (p.e. ácidos húmicos). Lo anterior puede explicar el hecho de que en el Andosol, con una fracción orgánica dominada por ácidos húmicos (Tabla 2), se observen dos aspectos relevantes: que el coeficiente de adsorción sea alto, indicando una alta afinidad por el herbicida y que la fuerza de adsorción sea alta, de lo cual da cuenta un coeficiente de desorción mayor que el coeficiente de adsorción (Tabla 4). En el Andosol, la cantidad de carbono orgánico disuelto (COD) es alta, lo que favorece la formación de complejos con el herbicida y con ello la presencia de atrazina en la fase liquida del suelo. De este modo, la concentración del herbicida al equilibrio, es el resultado de la competencia entre los diferentes adsorbentes en el sistema y el grado de reversibilidad de dichos procesos.

En el Andosol, la cantidad de carbono orgánico disuelto (COD) es alta, lo que favorece la formación de complejos con el herbicida y con ello la 
presencia de atrazina en la fase liquida del suelo. En la literatura se reporta que el COD en la solución del suelo interacciona con los herbicidas, lo que favorece su permanencia en la solución de suelo ya que puede interaccionar con la fracción soluble y formar complejos (Gamble et al., 1986; Devitt y Wiesner, 1998), lo que favorece el movimiento de atrazina en el suelo y puede explicar el transporte del herbicida a través del perfil de suelo (Masse et al., 1994), así como su presencia en el agua subterránea. Esto último ya ha sido reportado para la atrazina (Gamble et al., 1986; Devitt y Wiesner, 1998), en contraste, Spark y Swift (2002) observaron que la fracción de COD no tuvo un efecto significativo en la adsorción y transporte de atrazina en suelos cuyo contenido de COD era entre el 18 y el $25 \%$ del carbono orgánico total (COT) del suelo. En el Andosol estudiado, el COD equivale al $27 \%$ del carbono total, valor muy cercano al límite superior del reportado en la literatura. En la presente investigación no fue posible evaluar el efecto que el COD tuvo en la movilidad del herbicida, para ello habría sido necesario registrar los espectros UVvis de las soluciones de atrazina al equilibrio.

Un factor que puede explicar los valores bajos de $\mathrm{K}_{\mathrm{d}}$ en este Andosol respecto a los reportados en la literatura para otros suelos alofánicos, es la baja concentración del carbono orgánico del suelo (COS) del horizonte superficial de este suelo, respecto al contenido de este elemento en otros Andosoles: $100 \mathrm{~g} / \mathrm{kg}$ en los de la Isla de Java (Van-Ranst et al., 2002), $140 \mathrm{~g} / \mathrm{kg}$ en los del sur de Ecuador (Buytaert et al., 2002) y $95 \mathrm{~g} / \mathrm{kg}$ en suelos volcánicos de Chile (Escudey et al., 2004), lo cual sugerimos puede ser consecuencia del manejo agrícola intensivo al cual está expuesto el suelo estudiado, que incrementa la tasa de descomposición y lo hace susceptible a la remoción de la capa superficial por erosión (Viramontes et al., 2006).

El bajo contenido de COS puede deberse a dos factores favorecidos por el manejo agrícola al que es sometido, primero como parte de la práctica agrícola local, los residuos no son incorporados al final de la estación de cultivo, lo que significa que el suministro de COS no es renovado. De este modo, el horizonte orgánico pudo haberse perdido debido a la disminución de la fertilidad a lo largo de los años de uso agrícola. Un segundo factor puede ser la pérdida de carbono disuelto y particulado por escorrentía durante los eventos de erosión hídrica en épocas de lluvia. Al respecto, Prado et al. (2007) midieron la concentración de carbono particulado y disuelto en $45 \%$ de la escorrentía total durante la temporada de lluvias, y alrededor del $68 \%$ de la erosión durante un año. La pérdida de carbono particulado medido en 2003 fue de $416 \mathrm{~kg} \mathrm{C} / \mathrm{ha}$, que significa que la pérdida total de carbono particulado por escorrentía y erosión durante la época de lluvias fue de alrededor $600 \mathrm{~kg}$ de $\mathrm{C} / \mathrm{ha}$, lo cual representa entre el 15 al $20 \%$ de la concentración del carbono en el primer $\mathrm{cm}$ del perfil del suelo. Otro efecto negativo producido por el cambio de uso de suelo de bosque a agricultura de temporal, implica que la parcela esté desnuda durante algunos meses del año, y el suelo en superficie esté expuesto a la radiación solar, lo cual en un Andosol, provoca una agregación irreversible y con ello una redistribución del tamaño de las partículas (Nanzyo, 2002). La formación de partículas de tamaño $<0.25 \mathrm{~mm}$ (micro-agregados) en Andosoles de México, está relacionada con la disminución en el contenido de $\mathrm{MO}$, así como una reducción del contenido de retención de humedad (Meza-Pérez y Geissert-Kientz, 2006) que modifica la superficie específica y disminuye la capacidad de intercambio del suelo (Etchevers et al., 1986). De este modo, la pérdida de humedad del suelo y la consecuente formación de microagregados, aunado a la pronunciada pendiente $(>28 \%)$ con relieve convexo, provoca que en el sitio haya pérdida de suelo por erosión de tipo laminar. El conjunto de factores enlistados, han provocado una redistribución del suelo, lo cual se observa en las características mineralógicas de la capa superficial del perfil (Prado et al., 2007) y por ende, en la capacidad de adsorción del herbicida.

Otro factor importante que está relacionado con el manejo agrícola, y que puede influir sobre la capacidad de retención del herbicida, es el cam- 
bio de $\mathrm{pH}$ debido al uso de fertilizantes nitrogenados como urea, nitrato de amonio, Triple 17, entre otros. La disminución del pH del suelo bajo estas condiciones ha sido reportada por Shoji et al. (1996) en Andosoles japoneses. Lo anterior fue puesto de manifiesto por Prado et al. (2007), quienes estudiaron el perfil completo en el sitio y en donde los autores sugieren una disminución del $\mathrm{pH}$ de 6.1 - 6.5 a 5.5. La disminución del pH del suelo puede favorecer la adsorción de atrazina en sustancias húmicas. Al respecto Wang et al. (2011) observaron que la adsorción en estas es mayor a medida que disminuye el $\mathrm{pH}$ del suelo.

\subsection{ADSORGIÓN - DESORGIÓN DE ATRAZINA DE UN FEOZEM BAJO DIFERENTE MANEJO AGRÍCOLA: LABRANZA GERO Y LABRANZA GONVENGIONAL}

En los últimos años se han evaluado diversas prácticas agrícolas, bajo la premisa de modificar las existentes para disminuir los impactos al ambiente, incrementando la producción agrícola. Los dos extremos de esto son la agricultura convencional y la agricultura de conservación. En el primer caso se utiliza maquinaria para el laboreo, con adición de agroquímicos para compensar al suelo los nutrimentos perdidos en el cultivo anterior y en la mayoría de los casos, eliminando los residuos de la cosecha anterior. Por otro lado, la agricultura de conservación es cualquier sistema de manejo que incluye: una reducción importante del movimiento del suelo hasta eliminar esta práctica por completo, excepto por el disturbio que se provoca durante la siembra, la preservación de una cubierta orgánica permanente o semi permanente en el suelo y por último, la rotación de cultivos económicamente viables (FAO, 2002; Wall, 2006). Un tipo de agricultura de conservación es la labranza cero combinada con rotación de cultivos y retención de residuos, la cual ha mostrado mejorar las propiedades físicas, químicas y biológicas del suelo, además de incrementar la producción (Lichter et al., 2008; Govaerts et al., 2009). Por otro lado, la labranza cero requiere del uso de herbicidas en dosis más altas que la labranza convencional (Montoya et al., 2006; Dirk y Kurstjens, 2007). En ese contexto, resulta interesante evaluar el impacto en las propiedades del suelo por estos dos tipos de labranza, que están relacionadas con las interacciones que ocurren entre el herbicida y el suelo. El Feozem evaluado, mostró que tiene la capacidad de adsorber al herbicida atrazina, dependiendo del tipo de manejo agrícola. La Figura 3 muestra las isotermas de adsorción y desorción obtenidas en el Feozem bajo agricultura convencional y monocultivo de maíz (LC) y en el suelo bajo a.

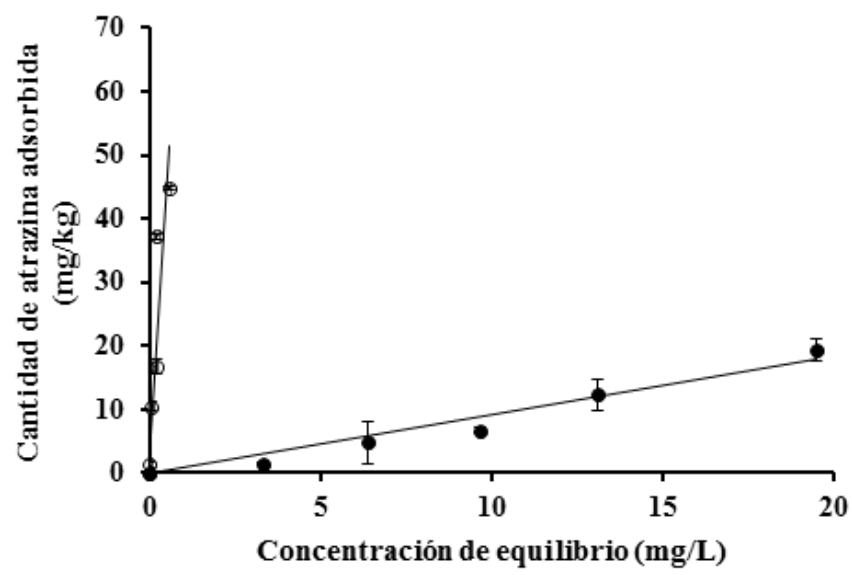

b.

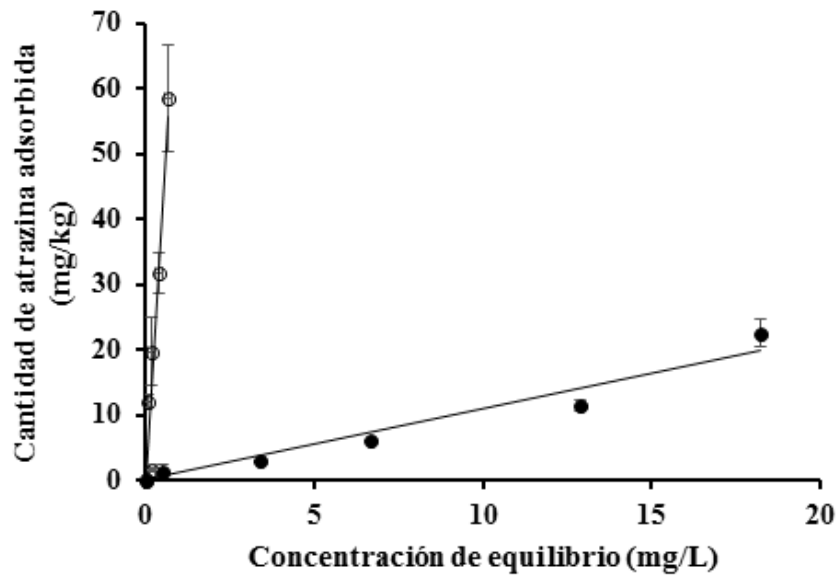

Figura 3 Isotermas de adsorción de atrazina (símbolos rellenos) y desorción (símbolos huecos), del suelo tipo Feozem de la estación experimental del CIMMYT. En $a$. Suelo de labranza convencional (LC) y en $b$. Suelo de labranza cero (LZ). Se indica la línea de ajuste lineal y las barras de desviación en ambos casos. 
labranza cero con rotación de cultivos y con residuos (LZ). La capacidad de adsorción de atrazina del suelo fue ligeramente más alta en el suelo bajo LZ que en el suelo bajo LC. En el caso de la desorción el resultado fue opuesto, el coeficiente de distribución de este proceso fue más alto en el suelo bajo LC. Los coeficientes de desorción fueron más de 70 veces mayores que los correspondientes de adsorción, resultado que indica que una vez que la atrazina es adsorbida en el suelo, presenta menor tendencia a la desorción.

Las parcelas estudiadas tenían establecidas alrededor de 20 años de establecidas al momento de realizar esta investigación, por lo que los suelos tenían un contenido similar de arcilla (Fuentes et al., 2009). Sin embargo, la diferencia en el manejo agrícola entre parcelas, resultó en un contenido diferente de COS en el horizonte superficial (Fuentes et al., 2012), por lo cual fue posible evaluar la capacidad de adsorción de atrazina del suelo con base en el contenido de carbono. Lo anterior considerando que la fracción orgánica del suelo, junto con las arcillas, son los principales adsorbentes del herbicida (Nicholls, 1988; Barriuso et al., 1992; Payá-Pérez et al., 1992; Dousset et al., 1994).

El contenido de COS fue mayor en el suelo LZ con rotación de cultivos $(28.5 \mathrm{~g} / \mathrm{kg})$ que en el suelo bajo LC (14.8 g/kg). Al respecto, Buyanovsky y Wagner (1998) observaron que la rotación de maíz y trigo fue más efectiva en la retención de carbono en el suelo que el monocultivo de maíz. De acuerdo con Govaerts et al. (2009), el efecto de la rotación del cultivo sobre la retención de carbono puede deberse al incremento de entrada de carbono debido a la producción intensificada o bien, debido al cambio de calidad de los residuos que ingresan al suelo. La diferencia en el contenido de COS entre los suelos, no provocó una diferencia importante en la capacidad de adsorción del herbicida. En el caso de la desorción, el haber obtenido un coeficiente de desorción más alto en el suelo con menor contenido de COS, puede atribuirse a la composición química y estado de degradación de los compuestos orgánicos del suelo, lo cual puede ser influido por las prácticas de manejo. Por ejemplo, la disrupción del suelo durante el arado intensivo (LG) promueve la descomposición al dejar los residuos de la cosecha y la MO del suelo protegida en agregados, expuesta a los factores abióticos (p.e. humedecimiento-secado, radiación UV) y bióticos (p.e. actividad microbiana) (Golchin et al., 1994; Allmaras et al., 2004), lo que disminuye la concentración de la MO en los suelos, en contraste a lo que ocurre en los sistemas de LZ donde los residuos son dejados en la superficie del suelo, la $\mathrm{MO}$ se descompone lentamente lo que permite una mayor acumulación de esta y específicamente de sustancias que pueden promover la retención del herbicida, es decir, las prácticas de manejo afectan la acumulación y composición química de la MO del suelo.

Esta condición podría explicar las diferencias en la caracterización de las sustancias húmicas de los suelos bajo diferente tipo de manejo. En el suelo LZ, la mayor parte del COS se encuentra como huminas $(\mathrm{LZ}=63 \%, \mathrm{LC}=46 \%$, ambos con relación al COS) es decir, en compuestos muy recalcitrantes, hidrófobos y de elevado peso molecular, que además pueden ser muy estables si se encuentran en interacción con compuestos minerales del suelo (Sánchez-González et al., 2017). Por otro lado, proporcionalmente hay más ácidos húmicos en LG que en LZ respecto al COS (43\% en LC y $24 \%$ en LZ), lo que nos indica que en los suelos bajo diferente manejo pueden suceder diferentes mecanismos predominantes de adsorción de la atrazina con el COS, por ejemplo en LC relacionado con la afinidad a los ácidos húmicos y en LZ relacionado a la afinidad con compuestos hidrófobos.

La acumulación de MO en el suelo guarda una estrecha relación con otras propiedades físicas del suelo tales como la porosidad y humedad, mismas que influyen directamente en la estructura y estabilidad de los agregados del suelo (Kay y Vanden Bygaart, 2002; Urbanek y Horn, 2006). Anteriormente en el sitio, Fuentes et al. (2012) pusieron de manifiesto que la captura de $\mathrm{C}$ ocurre de forma diferencial entre agregados de diferente tamaño. En este tipo de sistemas sería entonces interesante 
evaluar la adsorción - desorción del herbicida en los agregados de suelo, además del suelo tamizado como se realizó en este estudio.

\subsection{ADSORGIÓN - DESORGIÓN DE ATRAZINA EN VERTISOLES CON AGRICULTURA DE TEMPORAL Y BAJO RIEGO GON AGUA RESIDUAL}

Entre las prácticas agrícolas que se han desarrollado en los últimos años, se encuentra el uso de agua residual para el riego de cultivos en zonas agrícolas en donde el recurso hídrico es limitado. El riego con agua residual incorpora materia orgánica particulada y disuelta al sistema (Seol y Lee, 2000), así como también produce cambios en el $\mathrm{pH}$ y la composición electrolítica de la solución del suelo (Bond, 1998; Cameron et al., 2003). Tales factores definen el destino de la atrazina en el suelo notablemente, al modificar tanto cuantitativa como cualitativamente las características de la MO del suelo, tales como: concentración, biodegradabilidad, estabilidad (Fang et al., 1998; Barton y Karathanasis, 2003), polaridad y aromaticidad (Madhun et al., 1986; Thomsen et al., 2002), así como el contenido de fracciones hidrofóbicas (Ilani et al., 2005; Chefetz et al., 2006).

La región del Valle del Mezquital en el estado de Hidalgo, recibe desde hace más de 100 años las aguas residuales de la Ciudad de México para el riego de campos agrícolas. En este trabajo se evaluaron tres Vertisoles con diferente número de años bajo riego, un Vertisol de temporal (VT) y dos Vertisoles bajo agricultura intensiva regados con agua residual cruda, uno durante 56 años (V56) y otro durante 100 años (V100). Los tres Vertisoles se localizan en la parte más baja de un piedemonte volcánico, son suelos medianamente profundos, arcillosos, con alto contenido de $\mathrm{MO}$ y son altamente productivos.

Los datos obtenidos en los experimentos de adsorción y desorción de atrazina de los tres Vertisoles (VT, V56 y V100), fueron descritos adecuadamente con el modelo lineal (R2> 0.72) (Figuras 4a, 4c, 4e). Se observó una mayor adsorción en el suelo con 56 años bajo riego, y una dependencia entre el coeficiente de distribución y los tratamientos del suelo, siendo V56 > V100 > VT con valores de $\mathrm{K}_{\mathrm{d}}$ entre 11.0 y $3.8 \mathrm{~L} / \mathrm{kg}$ (Tabla 4). Se observó que la capacidad de adsorción obtenida tiene una relación inversa con el contenido del COS y el contenido de arcilla de los suelos evaluados (Tabla 2), es decir, a mayor contenido de carbono y de arcilla, menor capacidad de adsorción.

Similar a lo observado en los otros suelos evaluados (el Andosol y el Feozem), al comparar las isotermas de adsorción y desorción en los tres Vertisoles, se observó el fenómeno de histéresis entre las isotermas (Figura 4). Respecto a la desorción, en los tres suelos se obtuvieron coeficientes de distribución superiores a los obtenidos en la adsorción (Tabla 4), lo que indica que una vez que la atrazina es adsorbida en el suelo, esta es fuertemente retenida. Un resultado similar en Vertisoles fue observado por Naga et al. (2013). De manera similar a lo observado en la adsorción, el coeficiente de desorción más alto se obtuvo para el suelo V56, el valor de $\mathrm{K}_{\mathrm{d}}$ en la etapa de desorción tuvo el mismo comportamiento V56 > V100 > VT con valores entre 159 y $25 \mathrm{~L} / \mathrm{kg}$.

Las isotermas de adsorción obtenidas en los tres suelos sin $\mathrm{MO}$ se ajustaron al modelo lineal $\left(\mathrm{R}^{2}\right.$ $>0.75$ ) (Figuras 4b, 4d, 4f). En los tres casos, el valor de $\mathrm{K}_{\mathrm{d}}$ disminuyó respecto a los coeficientes obtenidos en los suelos con MO. Esta disminución fue de alrededor del $80 \%$ en los suelos regados con agua residual (V56 y V100), mientras que en el suelo de temporal fue del $50 \%$ (Tabla 4). La marcada disminución del valor de $\mathrm{K}_{\mathrm{d}}$ en los dos suelos regados con agua residual, permite suponer que en ambos la $\mathrm{MO}$ es el principal adsorbente de atrazina. No obstante, dados los valores de $\mathrm{K}_{\mathrm{d}}$ del suelo tratado se puede considerar que, en los tres suelos la fracción mineral también contribuye, aunque en menor proporción, en la adsorción del herbicida. La mayor o menor afinidad del herbicida con la MO podría explicarse conociendo la naturaleza de la MO, sin embargo esta información no se ha evaluado en los suelos estudiados. Con respecto a la desorción de atrazina en el suelo sin MO, se observa que ocurre el mismo fenómeno de histéresis que en el suelo con MO; así también, el 
a.

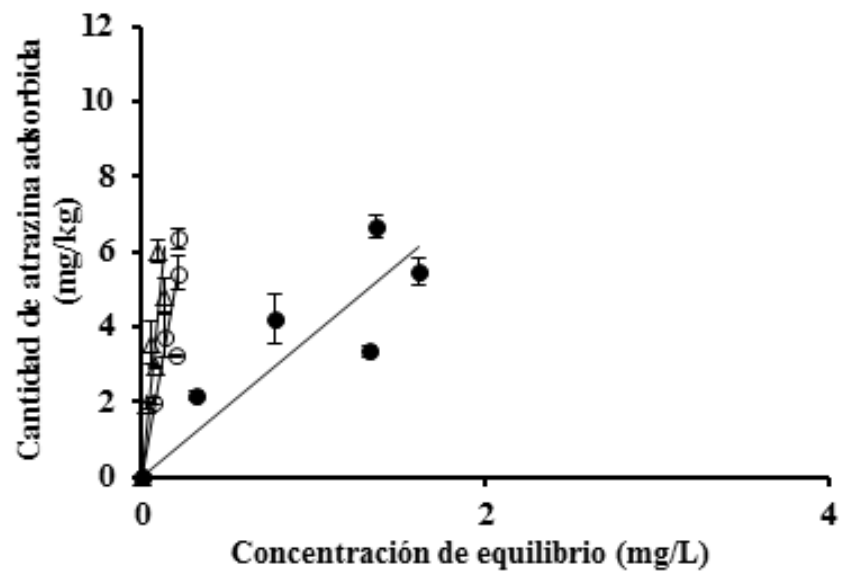

c.

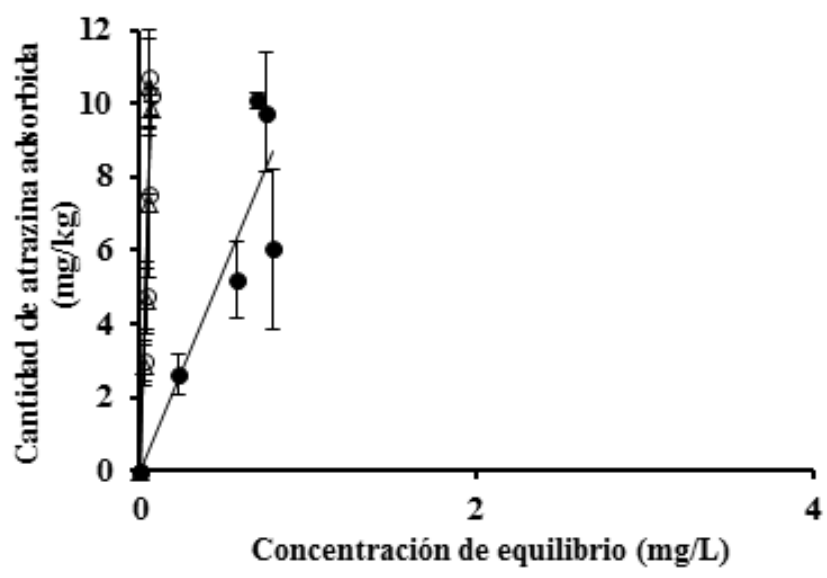

e.

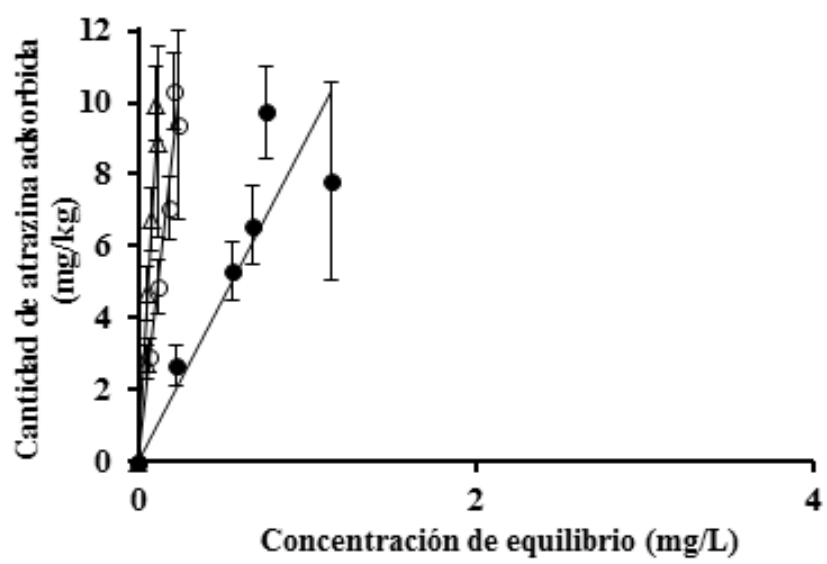

b.

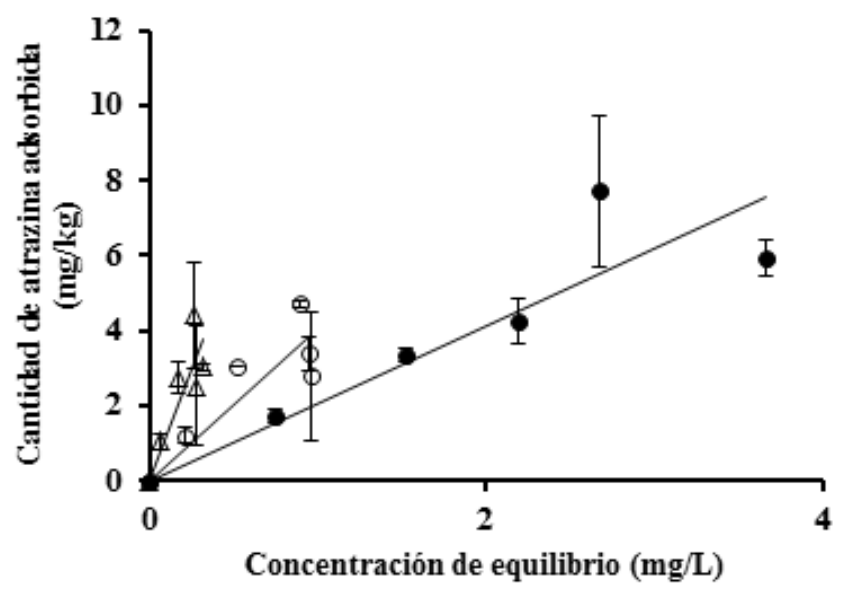

d.

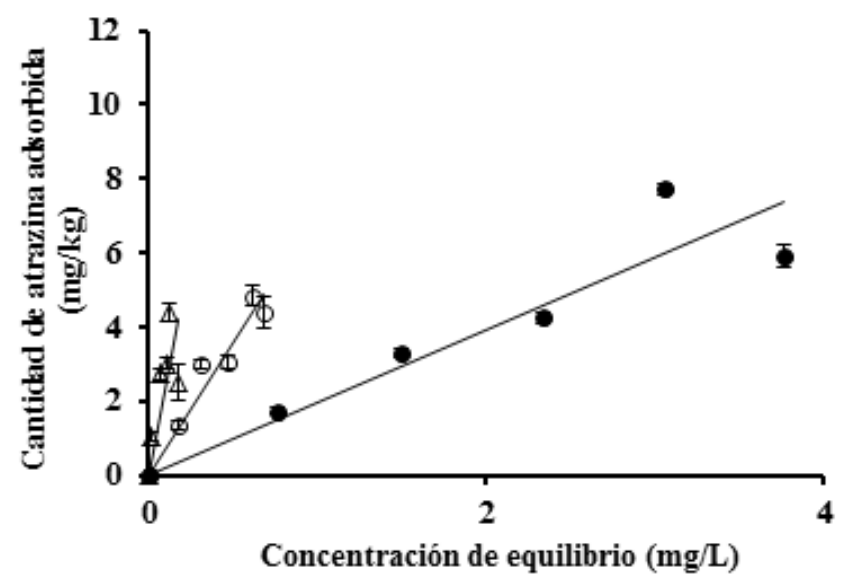

f.

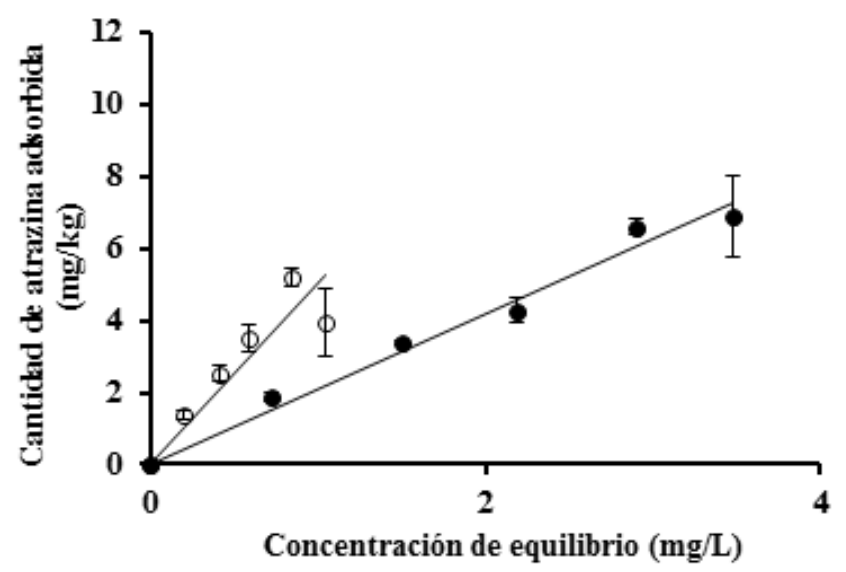

Figura 4 Isotermas de adsorción de atrazina (símbolos rellenos) y desorción (símbolos huecos) del suelo tipo Vertisol de las parcelas de cultivo en el estado de Hidalgo. En $\boldsymbol{a}$. Vertisol de la región de Ulapa bajo riego de temporal y en $\boldsymbol{b}$. Mismo Vertisol bajo tratamiento oxidativo con $\mathrm{NaOCl}$. En $c$. Vertisol de la región de Ulapa-Tetepango bajo riego con agua residual por 56 años y en $d$. Mismo Vertisol bajo tratamiento oxidativo con $\mathrm{NaOCl}$. En $e$. Vertisol de la región de Tetepango bajo riego con agua residual por 100 años y en $f$. Mismo Vertisol bajo tratamiento oxidativo con $\mathrm{NaOCl}$. Se indica la línea de ajuste lineal y barras de desviación. 
valor de $\mathrm{K}_{\mathrm{d}}$ de los tres suelos es mayor en la etapa de desorción que en la etapa de adsorción.

Una forma de evaluar el papel que juega la MO del suelo para la adsorción de atrazina, es normalizando el $\mathrm{K}_{\mathrm{d}}$ con el contenido de $\mathrm{CO}$, obteniéndose el valor del coeficiente $\mathrm{K}_{\mathrm{oc}}$. Los valores obtenidos fueron de 124, 660 y $339 \mathrm{~L} / \mathrm{kg}$ (Tabla 4) para VT, V56 y V100, respectivamente, mismos que se encuentran en el intervalo de los valores reportados para la atrazina por varios autores (Koskinen y Moorman, 1985; Clay y Koskinen, 1990; Moreau y Mouvet, 1998; Ben-Hur et al., 2003). El coeficiente $\mathrm{K}_{\mathrm{oc}}$ asume que el principal adsorbente de atrazina es la fracción orgánica del suelo y para que esto sea válido, la relación entre el contenido de arcilla y el contenido de $\mathrm{CO}$ del suelo debe ser inferior a 30 (Ben-Hur et al., 2003), lo cual se cumple en los tres suelos (Tabla 4).

Los resultados obtenidos de los diferentes experimentos de adsorción - desorción mencionados, permiten concluir que en los Vertisoles estudiados, tanto la MO del suelo como la fracción mineral participan en la capacidad de adsorción del herbicida. $\mathrm{Al}$ parecer, la fracción orgánica es el principal adsorbente en los suelos bajo riego con agua residual V56 y V100, mientras que en el suelo VT las arcillas tienen el papel principal en la capacidad de adsorción del suelo. La adsorción en arcillas tipo montmorillonita, más que en la fracción orgánica de Vertisoles, fue reportada por Sheng et al. (2001) y Hiller et al. (2008). Celis et al. (1997) reportan que las arcillas del tipo montmorillonita pueden contribuir de forma importante en la capacidad de adsorción de atrazina, siendo este tipo de arcilla la dominante en los suelos estudiados (Siebe, 1994). La desorción del herbicida desde los suelos sin MO se entiende como la desorción desde la fracción mineral, en ese escenario el hecho de haber obtenido valores de $\mathrm{K}_{\mathrm{d}}$ de desorción mucho menores que los obtenidos en el suelo con $\mathrm{MO}$, podría indicar una menor reactividad de los sitios superficiales de la montmorillonita respecto de los modos de interacción de la MO. De la misma manera, DeSutter et al. (2003) reportan que existe mayor desorción del herbicida en suelos con menor contenido de carbono orgánico. Un dato interesante que puede desprenderse de este análisis es el hecho de que, aparentemente, el número de años bajo manejo agrícola y riego con agua residual no han afectado la capacidad de la fracción mineral de adsorber atrazina. Esto puede deberse a que probablemente el riego con agua residual no altera la fracción mineral del suelo o bien, en caso contrario, a que el efecto que tiene en ellas no provoca ningún cambio que modifique la adsorción del herbicida.

Varios autores reportan que el riego con agua residual cambia las propiedades del suelo al incorporar una gran cantidad de $\mathrm{MO}$ al sistema (Müller et al., 2007; Sánchez, 2008; Travis et al., 2010), produciendo incrementos en el contenido de carbono orgánico total y disuelto. Sin embargo, en los suelos estudiados, el mayor contenido de COS se observa en el suelo de temporal (VT), no obstante, la mayor capacidad de adsorción de atrazina se observó en los suelos regados con agua residual (V56 y V100) con un menor contenido de COS que el del suelo de temporal. Entre los suelos regados con agua residual, el suelo con 100 años bajo riego tiene más COS que el suelo con 56 años bajo riego. De este modo, la mayor capacidad de adsorción de atrazina del Vertisol con menor contenido de carbono orgánico podría explicarse considerando la calidad del mismo. Al respecto, en estudios realizados en el Valle del Mezquital, Siebe et al. (2016) reportan que el uso de agua residual para el riego de zonas agrícolas, ha incrementado el contenido de la MO del suelo en más de un $60 \%$ durante los primeros 40 años, hasta que se alcanza un nuevo equilibrio entre el aumento de la producción de biomasa y su descomposición. De acuerdo con estos autores, factores como el manejo agrícola y el número de años bajo riego con agua residual aumentan la hidrofobicidad. En suelos del Valle del Mezquital, Sánchez-González et al. (2012) reportaron que a mayor número de años bajo riego la $\mathrm{MO}$ del suelo es menos aromática, lo que resulta en una disminución de la afinidad por el herbicida.

El COD del suelo también es un factor importante 
al estudiar el destino de la atrazina en el suelo, esto debido a que el COD funciona como el vector que moviliza al herbicida hacia estratos más profundos (Gamble et al., 1986; Devitt y Wiesner, 1998) y además puede competir con la atrazina por los sitios de sorción disponibles. Sánchez (2008) reporta que en los suelos del Valle del Mezquital, el contenido de COD del suelo ha aumentado con el número de años bajo riego, lo que implicaría que la movilidad del herbicida aumente en suelos con mayor número de años bajo riego. Esto podría explicar el resultado observado: una mayor movilidad y menor capacidad de adsorción en el suelo V100 que en el suelo V56. Sin embargo, los mismos autores reportan que la fracción lábil del COD también aumenta con el número de años bajo riego, de tal forma que se espera que el COD no sea un factor que favorezca la movilidad del herbicida, es decir, se espera que con el aumento del número de años bajo riego prevalezca la adsorción sobre la movilidad del herbicida.

$\mathrm{Al}$ evaluar el grado de histéresis, se observa que fue mayor en los suelos V56 y V100 en los cuales la adsorción de atrazina se asocia más a la fracción orgánica que a la fracción mineral del suelo. En un análisis de la histéresis entre la adsorción y la desorción de pesticidas no iónicos como la atrazina, Bailey y White (1964) y Harper, (1988) observaron que estos son retenidos sobre las superficies de minerales del suelo mediante interacciones electrostáticas débiles (fisisorción), mientras que ambos tipos de interacciones físicas y químicas, pueden ocurrir en los enlaces entre los pesticidas no iónicos y la MO del suelo (Sharom y Stephenson, 1976; Peck et al., 1980). Esto puede entonces explicar el hecho de haber obtenido una menor histéresis en el suelo VT, en el cual la adsorción de atrazina se asocia a las arcillas del suelo. En este sentido, varios autores han reportado las interacciones relativamente débiles entre pesticidas no iónicos y los minerales del suelo (Bailey y White 1964; Harper, 1988; Gilchrist et al., 1993).

\section{Conclusiones}

La capacidad de adsorción - desorción de la atrazina en los suelos estudiados está en función del contenido y composición química de la $\mathrm{MO}$ del suelo, la cual se ve afectada con las prácticas de manejo agrícola del suelo en cada localidad, con lo que es posible decir que el manejo afecta la capacidad del suelo de retener al herbicida.

En el Andosol con agricultura de temporal, la adsorción de atrazina fue mayor que en los Feozems y que en los Vertisoles estudiados. El mayor contenido de COS en este suelo explica este comportamiento. Los suelos ándicos tienen generalmente altos contenidos de carbono orgánico, sin embargo, la disminución de este en el suelo estudiado, como resultado de la deforestación del bosque y el cambio de uso de suelo para agricultura de temporal, disminuye el contenido de carbono y entonces, disminuye la capacidad de retención del herbicida en comparación con los valores reportados en la literatura para suelos ándicos. Además, la deshidratación del suelo superficial al estar expuesto a la radiación solar debido a la práctica de dejar desnudas las parcelas, provoca que se formen agregados irreversibles debido al contenido de minerales alofánicos, modificando la distribución del tamaño de las partículas y reduciendo con ello la superficie especifica del suelo, que se traduce en una disminución de la superficie de adsorción del herbicida. En los Feozems, la agricultura con labranza cero favorece la acumulación de la MO del suelo ya que es un sistema de reciclaje de residuos de cosecha, sin embargo, en la labranza convencional (LC) se favorece la mineralización de la fracción orgánica, lo que provoca una menor reversibilidad de la adsorción del herbicida. Por otro lado, la labranza convencional favorece la aireación del suelo, lo cual en teoría, pudiera favorecer a su vez la degradación de la atrazina disminuyendo con ello el riesgo de acumulación del herbicida.

La actividad agrícola permanente en zonas semiáridas es posible gracias a la disponibilidad de agua residual. El riego con agua residual favorece 
la acumulación de $\mathrm{MO}$ en el suelo, sin embargo, no solo el contenido de la MO del suelo cambia, sino también las características de este componente del suelo. Siendo que la adsorción del herbicida en el suelo está relacionada tanto con el contenido como con la calidad de la MO del suelo, la capacidad de adsorción del herbicida no se incrementa de manera directa con el número de años bajo riego. Algo similar ocurre con el COD aportado por el agua residual, el cual facilita el transporte del herbicida desde la superficie del suelo a estratos más profundos. El COD también aumenta con el número de años bajo riego, sin embargo, se va haciendo menos afín al herbicida. En los Vertisoles estudiados, la retención del herbicida en la fracción mineral también fue importante, sobre todo en el suelo de temporal. No obstante, el análisis realizado puso en evidencia que la adsorción de atrazina ocurre de manera más intensa en la fracción orgánica del suelo.

Uno de los servicios ecosistémicos que nos ofrecen los suelos es su función como amortiguador de contaminantes previniendo su llegada al acuífero.

Tal función implica la retención y/o degradación de los contaminantes en la zona vadosa debido a la influencia de procesos físicos, químicos y biológicos. El uso de compuestos orgánicos en la agricultura, como el herbicida atrazina estudiado en este trabajo, puede modificar la estructura funcional de la comunidad microbiana, afectando con ello las funciones biológicas de los suelos (Martin-Laurent, 2006). Aunado a lo anterior, el manejo no adecuado del suelo promueve la modificación de las fracciones mineral y orgánica de los suelos, modificando a su vez la capacidad de adsorción/ desorción de contaminantes, lo cual ha sido puesto en evidencia en este manuscrito.

Los resultados generados en este trabajo suman conocimiento sobre el efecto que las prácticas humanas tienen en la función amortiguadora del suelo en relación al herbicida atrazina en varios sitios de México, contribuyendo de esta manera a una mejor comprensión de los cambios en los ciclos naturales durante el Antropoceno.

\section{Agradecimientos}

Maricarmen Salazar Ledesma (212693) agradece a CONACyT por su beca de Doctorado. Los autores agradecen a la Mtra. Kumiko Shimada y al Biol. René Alcalá por su apoyo en la realización de los análisis físico-químicos de los suelos, y al Laboratorio Universitario de Nanotecnología Ambiental (LUNA) por realizar algunos de los análisis. Este trabajo fue apoyado por el CONACyT a través de los proyectos 220489 y 194017 , el proyecto IN105715 DGAPA-UNAM e ICyTDF/324/2011. Los autores reconocen a la familia Cornejo-Oviedo del Rancho Las Palmas por su valiosa contribución para la realización de este trabajo.

\section{Referencias}

Allmaras, R.R., Linden, D.R., Clapp, C.E., 2004, Corn-residue transformations into root and soil carbon as related to nitrogen, tillage, and stover management: Soil Science Society of America Journal, 68, 1366-1375.

Arienzo, M., Buondonno, A., 1993, Adsorption of paraquat by Terra Rossa soil and model soil aggregates: Toxicological and Environmental Chemistry, 39, 193-199.

Bailey, G.W., White, J.L., 1964, Review of adsorption and desorption of organic pesticides by soil colloids with implications concerning pesticide bio-activity: Journal of Agricultural and Food Chemistry, 12(4), 324-332.

Barriuso, E., Baer, U., Calvet, R., 1992, Dissolved organic matter and adsorption-desorption of dimefuron, atrazine and carbetamide by soils: Journal of Environmental Quality, 21, 359-367.

Barton, C.D., Karathanasis, A.D., 2003, Influence of soil colloids on the migration of atrazine and zinc through large monoliths: Water, Air, and Soil Pollution, 143, 3-21. 
Baskaran, S., Bolan, M.S., Rahman, A., Tillman, R.W., 1996, Pesticide sorption by allophanic and non-allophanic soils of New Zealand: New Zealand Journal of Agricultural Research, 39, 297-310.

Bekbolet, M., Yenigun, O., Yucel, I., 1999, Sorption studies of 2,4-D on selected soils: Water, Air, and Soil Pollution, 111, 75-88.

Ben-Hur, M., Letey, J., Farmer, W.J., Williams, G.F., Nelson, S.D., 2003, Soluble and solid organic matter effects on atrazine adsorption in cultivated soils: Soil Science Society of America Journal, 67, 1140-1146.

Blum, W.E.H., Eswaran, H., 2004, Soils and sediments in the Anthropocene: Journal of Soils and Sediments, 4(2), 71.

Bond, W.J., 1998, Effluent irrigation - an environmental challenge for soil science: Australian Journal of Soil Research, 36, 543-555.

Buyanovsky, G.A., Wagner, G.H., 1998, Changing role of cultivated land in the global carbon cycle: Biology and Fertility of Soils, 27, 242-245.

Buytaert, W., Deckers, J., Dercon, G., Bievre, B.D., Govers, J.P., 2002, Impact of land use changes on the hydrological properties of volcanic ash soils in South Ecuador: Soil Use and Management, 18, 94-100.

Cameron, K.G., Di, H.J., Anwar, M.R., Russell, J.M., Barnett, J.W., 2003, The "critical" ESP value: does it change with land application of dairy factory effluent?: New Zealand Journal of Agricultural Research, 46, 147-154.

Cejudo-Espinosa, E., Velázquez-Zepeda, A., Rodríguez-Vázquez, R., 2009, Adsorción de atrazina en secciones circulares de raíces de tres plantas de humedales: Revista Mexicana de Ingeniería Química, 8(1), 35-39.

Celis, R., Cornejo, J., Hermosín, M.C., Koskinen, W.C., 1997, Sorption-desorption of atrazine and simazine by model soil colloidal components: Soil Science Society of America Journal, 61(2), 436-443.
Chefetz, B., Ilani, T., Schulz, E., Chorover, J., Han, M.Y., Park K.H., Dockko, S., 2006, Wastewater dissolved organic matter: characteristics and sorptive capabilities: Water Science and Technology, 53, 51-57.

Clay, S.A., Koskinen, W.C., 1990, Adsorption and desorption of atrazine, hydroxyatrazine, and S-glutathione atrazine on two soils: Weed Science, 38, 262-266.

Close, M.E., Magesan, G.N., Lee, R., Stewart, M.K., Hadfield, J.C., 2003, Field study of pesticides leaching in an allophanic soil in New Zealand. Experiments results: Australian Journal of Soil Research, 41, 809-824.

Comisión de las Comunidades Europeas, (COM), 2002, 170 final, Hacia una estrategia temática para la protección del suelo, Bruselas, Bélgica. Consejo de la Cuenca del Valle de México (GGVM), 2016, Comisión de Guenca Valle de Bravo-Amanalco, disponible en < http:// cuencavalledemexico.com/ >, consultado 15 de octubre de 2016.

Cortes, V., 2016, Determinación simultánea de hidroxiatrazina, desetilatrazina y atrazina por medio de CLAR-UV-DAD en muestras de agua de las Lagunas de Montebello: México, D.F., Universidad Nacional Autónoma de México, Facultad de Química, Tesis de Licenciatura, 58 p.

De Jonge, R.J., Breure, A.M., van Andel, J.G., 1996, Reversibility of adsorption of aromatic compounds onto powdered activated carbon (PAC): Water Research, 30(4), 883-892.

DeSutter, T.H., Clay, S.A., Clay, D.E., 2003, Atrazine sorption and desorption as affected by aggregate size, particle size, and soil type: Weed Science, 51, 456-462.

Devitt, E.C., Wiesner, M.R., 1998, Dialysis investigations of atrazine organic matter interactions and the role of a divalent metal: Environmental Science and Technology, 32, 232-237. 
Dirk, A., Kurstjens, G., 2007, Precise tillage systems for enhanced non-chemical weed management: Soil and Tillage Research, 97, 293-305.

Domagalski,J.L., Dubrovsky, N.M., 1992, Pesticide residues in ground water of the San Joaquin Valley, California. Journal of Hydrology, 130, 299-338.

Dousset, S., Mouvet, C., Schiavon, M., 1994, Sorption of terbuthylazine and atrazine in relation to the physico-chemical properties of three soils: Chemosphere, 28 (3), 467-476.

Dragun, J., 1998, Adsorption and mobility of organic chemicals, en Dragun, J., (ed.), The Soil Chemistry of Hazardous Materials, 2nd. Ed., Massachusetts, USA, Amherst Scientific Publishers.

Dutta, A., Mandal, A., Manna, S., Singh, S. B., Berns, A.E., Singh, N., 2015, Effect of organic carbon chemistry on sorption of atrazine and metsulfuron-methyl as determined by (13) C-NMR and IR spectroscopy: Environmental Monitoring and Assessment, 187(10), 1-12.

El-Bestawy, E., Sabir, J., Mansy, A.H., Zabermawi, N., 2013, Isolation, identification and aclimatization of atrazine-resistant soil bacteria: Annals of Agricultural Science, 58(2), 119-130.

Escudey, M., Forster, J.E., Galindo, G., 2004, Relevance of organic matter in some chemical and physical characteristics of volcanic ash-derived soils: Communications in Soil Science and Plant Analysis, 35(5-6), 781-797.

Etchevers, J., Oleschko, K., Cruz, L., Hidalgo, C., 1986, Complex nature of the mechanical elements in Andosols from Mexico (resume), en XIII Congress of the International Society of Soil Science, Hamburgo, Germany.

European Parliament, 1998, Council directive 98/83/EG of 3 November 1998 on the quality of water intended for human consumption. Official Journal of the European Communities, L 330/32.
Fang, F., Kanan, S., Patterson, H.H., Cronan, C.S., 1998, A spectrofluorimetric study of the binding of carbofuran, carbaryl and aldicarb with dissolved organic matter: Analytica Chimica Acta, 373, 139-151.

Food and Agriculture Organization (FAO), International Soil Reference and Information Centre (ISRIC), International Soil Science Society (ISSS), 1998, World Reference Base for Soil Resources (WRB): World Soil Resources, 84, FAO, Roma, Italia.

Food and Agriculture Organization (FAO), 2002, Conservation agriculture when agriculture is profitable and sustainable, in FAO Land and water digital media series 18. Rome, Italy.

Food and Agriculture Organizaton (FAO), 2011, El estado mundial de la agricultura y la alimentación 2010-2011, Roma, Italia, disponible en <http://www.fao. org/docrep/013/i2050s/i2050s00.htm>, consultado 30 de septiembre de 2016.

Food and Agriculture Organization Corporate Statistical Database

(FAOSTAT) 2011. Disponible en: http://faostat. fao.org/site/423/DesktopDefault. aspx? PageID=423\#ancor.

Fuentes, M., Govaerts, B., De Leon, F., Hidalgo, C., Sayre, K.D., Etchevers, J., Dendooven, L., 2009, Fourteen years of applying zero and conventional tillage, crop rotation and residue management systems and its effect on physical and chemical soil quality: European Journal of Agronomy, 30(3), 228-237.

Fuentes, M., Hidalgo, C., Etchevers, J., De León, F., Guerrero, A., Dendooven, L., Verhulst, N., Govaerts, B., 2012, Conservation agriculture, increased organic carbon in the top-soil macro-aggregates and reduced soil $\mathrm{CO} 2$ emissions: Plant and Soil, 355, 183-197.

Gamble, D.S., Haniff, M.I., Zienius, R.H., 1986, Solution phase complexing of atrazine by fulvic acid: A batch ultrafiltration technique: Analytical Chemistry, 58, 727-731.

Garrido-Pérez, A., Cotler-Ávalos, H., EnríquezGuadarrama, C., 2006, Geomorfología y 
suelos, en Cotler-Ávalos, H., Mazari-Hiriart, M., de Anda-Sánchez, J. (eds), Atlas de la Cuenca Lerma-Chapala, construyendo una visión conjunta, $1^{a}$ ed., México D.F., INE-SEMARNAT, disponible en <http:// agua.org.mx/biblioteca/atlas-de-la-cuencalerma-chapala-construyendo-una-visionconjunta/>, consultado 10 de octubre de 2016.

Gilchrist, G.F.R., Gamble, D.S., Kodama, H., Khan, S.U., 1993, Atrazine interactions with clay minerals: Kinetics and equilibria of sorption: Journal of Agricultural and Food Chemistry, 41(10), 1748-1755.

Golchin, A., Oades, J.M., Skjemstad, J.O., Clarke, P., 1994, Study of free and occluded particulate organic matter in soils by solid state 13C Cp/MAS NMR spectroscopy and scanning electron microscopy: Australian Journal of Soil Research, 32, 285-309.

González-Márquez, L.C., Hansen, A.M., 2009, Adsorción y mineralización de atrazina y relación con parámetros de suelos del DR 063 Guasave, Sinaloa: Revista Mexicana de Ciencias Geológicas Sinaloa, 26, 587-599.

Govaerts, B., Sayre, K.D., Goudeseune, B., De Corte, P., Lichter, K., Dendooven, L., Deckers, J., 2009, Conservation agriculture as a sustainable option for the central Mexican highlands: Soil and Tillage Research, 103, 222-230.

Harper, S.S., 1988, Sorption of metribuzin in surface and subsurface soils of the Mississippi delta region: Weed Science, 36(1), 84-89.

Hayes, M.H.B., 1970, Adsorption of triazine herbicides on soil organic matter including a short review on soil organic matter chemistry: Residue Reviews, 32, 131-174.

Hernández-Antonio, A., Hansen, A.M., 2011, Uso de plaguicidas en dos zonas agrícolas de México y evaluación de la contaminación de agua y sedimentos: Revista Internacional de Contaminación Ambiental, México, 27, 115-127.
Hiller, E., Krascsenits, Z., Čerňanský, S., 2008, Sorption of acetochlor, atrazine, 2, 4-D, chlorotoluron, MCPA, and trifluralin in six soils from Slovakia: Bulletin of Environmental Contamination and Toxicology, 80(5), 412-416.

Huber, M., Knutti, R., 2012, Anthropogenic and natural warming inferred from changes in Earth's energy balance: Nature Geoscience, 2012, 5(1), 31-36.

Ilani, T., Schulz, E., Chefetz, B., 2005, Interactions of organic compounds with wastewater dissolved organic matter: role of hydrophobic fractions: Journal of Environmental Quality, 34, 552-562.

Instituto Nacional de Estadística Geografía e Informática (INEGI), 1999, Superficie continental e insular del territorio nacional. México, D.F.

International Union of Soil Sciences (IUSS) Working Group, 2006, World reference base for soil resources 2006. World Soil Resources Reports No. 103, FAO, Roma, Italia.

Jarvis, N.J., Jansson, P.E., Dik, P.E., Messing, I., 1991, Modelling water and solute transport in macroporous soils I. Model description and sensitivity analysis: European Journal of Soil Science, 42, 59-70.

Jenks, B.M., Roeth, F.W., Martin, A.R., McCallister, D.L., 1998, Influence of surface and subsurface soil properties on atrazine sorption and degradation: Weed Science, 46, 132-138.

Kay, B.D., Vanden Bygaart, A.J., 2002, Conservation tillage and depth stratification of porosity and soil organic matter: Soil and Tillage Research, 66, 107-1 18.

Kibblewhite, M.G., Ritz, K., Swift, M.J., 2008, Soil health in agricultural systems: Philosophical Transactions of the Royal Society B, 363, 685-701.

Kim, S.H., Fan, M., Prasher, S.O., Patel, R.M., Hussain, S.A., 2011, Fate and transport of atrazine in a sandy soil in the presence of 
antibiotics in poultry manures: Agricultural Water Management, 98, 653-660.

Kögel-Knabner, I., 2002, The macromolecular organic composition of plant and microbial residues as input to soil organic matter: Soil Biology and Biochemistry, 34, 139-162.

Koskinen, W.C., Moorman, E.E., 1985, Factors affecting triazine and substituted-urea adsorption in soil (resume), en Weed Science Society of America, Illinois, USA.

Kulikova, N.A., Perminova, I.V., 2002, Binding of atrazine to humic substances from soil, peat and coal related to their structure: Environmental Science and Technology, 36(17), 3720-3724.

Lal, R., 1993, Tillage effects on soil degradation, soil resilience, soil quality, and sustainability: Soil and tillage Research, 27(1-4), 1-8.

Laursen, A.E., Carlton, R.G., 1999, Responses to atrazine of respiration, nitrification, and denitrification in stream sediments measured with oxygen and nitrate microelectrodes: FEMS Microbiology Ecology, 29(3), 229-240.

LeBaron, H.M., McFarland, J.E., Burnside, O.G., 2008, The Triazine Herbicides: A Milestone in the Development of Weed Control Technology, en LeBaron, H.M., McFarland, J.E., Burnside, O.C. (eds.), The Triazine Herbicides, 50 Years Revolutionizing Agriculture, 1st. ed., San Diego, CA, Elsevier, $1-12$.

Lerch, R.N., Sadler, E.J., Sudduth, K.A., Baffaut, C., Kitchen N.R., 2011, Herbicide transport in Goodwater Creek experimental watershed: I. Long-term research on atrazine: Journal of the American Water Resources Association 47, 209-223.

Lichter, K., Govaerts, B., Six, J., Sayre, K.D., Deckers, J., Dendooven, L., 2008, Aggregation and $\mathrm{C}$ and $\mathrm{N}$ contents of soil organic matter fractions in the permanent raised-bed planting system in the Highlands of Central Mexico: Plant and Soil, 305, 237-252.
Loiseau, L., Barriuso, E., 2002, Characterization of the atrazine's bound (non-extractable) residues using fractionation techniques for soil organic matter: Environmental Science and Technology, 36, 683-689.

Madhun, Y.A., Young, J.L., Fred, V.H., 1986, Binding of herbicides by water-soluble organic materials from soil: Journal of Environmental Quality, 15, 64-68.

Martin-Laurent, F., Barrès, B., Wagschal, I., Piutti, S., Devers, M., Soulas, G., Philippot, L., 2006, Impact of the Maize Rhizosphere on the Genetic Structure, the Diversity and the Atrazine-degrading Gene Composition of Cultivable Atrazine-degrading Communities. Impact of the Maize Rhizosphere on the Genetic Structure, the Diversity and the Atrazine-degrading Gene Composition of Cultivable Atrazine-degrading Communities. Plant Soil 282:99-115.

Masse, L., Prasher, S.O., Khan, S.U., Arjoon, D.S., Barrington, S., 1994, Leaching of metolachlor, atrazine, and atrazine metabolites into groundwater: Transactions of the ASAE, 37, 801-806.

Meza-Pérez, E., Geissert-Kientz, D., 2006, Estabilidad de estructura en Andisoles de uso forestal y cultivados: Terra Latinoamericana, 24 (2), 163-170.

Miller, M.S., Doxtater, K.G., 1995, Atrazine impacts of shortgrass prairie micro-cosms: Journal of Range Management, 48, 298-306.

Montaño, J.M., 2013, Elucidación de los mecanismos de movilidad y retención de atrazina e hidroxiatrazina en suelos por medio de GLAR-DAD: México, D.F., Universidad Nacional Autónoma de México, Facultad de Química, Tesis de Licenciatura, 84p.

Montoya, J.C., Costa, J.L., Liedl, R., Bedmar, F., Daniel, P., 2006, Effects of soil type and tillage practice on atrazine transport through intact soil cores: Geoderma, 137, 161-173.

Moreau, C., Mouvet, C., 1998, Adsorption and desorption of atrazine, deethylatrazine, and hydroxyatrazine by soil components: Journal 
of Environmental Quality, 27(1), 46-53.

Mudhoo, A., Garg, V.K., 2011, Sorption, transport and transformation of atrazine in soils, minerals and composts: A review: Pedosphere, 21(1), 11-25.

Müller, K., Magesan, G.N., Bolan N.S., 2007, A critical review of the influence of effluent irrigation on the fate of pesticides in soil: Agriculture, Ecosystems and Environment, 120, 93-116.

Myers, S.S., Patz, J.A., 2009, Emerging threats to human health from global environmental change. Annual Review of Environmental Resources, 34(1), 223-252.

Naga, M.K.V., Chandrasekhar, R.P., Subba, R.M., Prathima, T., Giridhar, V., 2013, Adsorption-desorption of atrazine on vertisols and alfisols: Indian Journal of Weed Science, 45(4), 273-277.

Nanzyo, M., 2002, Unique properties of volcanic ash soils: Global Environmental Research, 6 (2), 99-1 12.

Nicholls, P.H., 1988, Factors influencing the entry of pesticides into soil water: Pesticide Science, 22, 123-137.

Nieto-Samaniego, A.F., Alaniz-Álvarez, S.A., Camprubí, A., 2005, La Mesa Central de México, estratigrafía, estructura y evolución tectónica cenozoica: Boletín de la Sociedad Geológica Mexicana, Volumen Conmemorativo de Bicentenario, Temas Selectos de la Geología Mexicana, Tomo LVII, 3, 285-318.

Organization for Economic Co-operation and Development (OECD), 2002, Annual report, disponible en <https:// www.oecd. org/about/2080175.pdf $>$, consultado 8 de septiembre de 2016.

Parfitt, R.L., 1990, Allophane in New Zealand - a review: Australian Journal of Soil Research, 28, 343-360.

Payá-Pérez, A.B., Cortés, A., Sala, M.N., Larsen, B., 1992, Organic matter fractions controlling the sorption of atrazine in sandy soils: Chemosphere, 25(6), 887-898.
Peck, D.E., Corwin, D.L., Farmer, W.J., 1980, Adsorption-desorption of diuron by freshwater sediments: Journal of Environmental Quality, 9 (1), 101-106.

Pimm, S.L., Russell, G.J., Gittleman, J.L., Brooks, T.M. 1995. The Future of Biodiversity. Science 269 (5222), 347-350.

Prado, B., Duwig, C., Hidalgo, C., Gómez, D., Yee, H., Prat, C., Esteves, M., Etchevers, J.D., 2007, Characterization, functioning and classification of two volcanic soil profiles under different land uses in Central Mexico: Geoderma, 139, 300-313.

Prado, B., Duwig, C., Hidalgo, C., Müller, K., Mora, L., Raymundo, E., Etchevers, J.D., 2014a, Transport, sorption and degradation of atrazine in two clay soils from Mexico: Andosol and Vertisol: Geoderma, 233-234, 628-639.

Prado, B., Fuentes, M., Verhulst, N., Govaerts, B., De León, F., Zamora, O., 2014b, Fate of atrazine in a soil under different agronomic management practices: Journal of Environmental Science and Health, Part B, 49, 844-855.

Riise, G., Eklo, O.M., Lode, O., Pettersen, M.N., 1994, Mobility of atrazine and tribenuron methyl in the soil water system lysimeter experiments: Norwegian Journal of Agricultural Science Supplement, 13, 31-41.

Ross, M., Goberna, M., Moreno, J.L., Hernández, T., García, C., Insam, H., 2006, Molecular and physiological bacterial diversity of a semiarid soil contaminated with different levels of formulated atrazine: Applied Soil Ecology, 34, 93-102.

Roy, W.R., Krapac, I.C., Chou, S.F.J., Griffin, R.A., 1991, Batch-type procedures for estimating soil adsorption of chemicals: EPA/530-SW-87-006-F, Illinois, U.S.A., U.S. Environmental Protection Agency.

Salazar-López, N., Aldana-Madrid, M., 2011, Herbicida glifosato: Usos, toxicidad y regulación: Revista de Ciencias Biológicas y de la Salud de la Universidad de Sonora, 
$13(2), 23-28$.

Sánchez, A., 2008, Cambios en la calidad de la materia orgánica disuelta en suelos regados con agua residual: México, D.F., Universidad Nacional Autónoma de México, Instituto de Geología, Tesis de Maestría.

Sánchez-González, A., Chapela, M., Germán, E., Fuentes-García, R., del Río-Portilla, F., Siebe, C., 2012, Cambios en el almacén de carbono del suelo y su calidad a través de la historia de uso en el Valle del Mezquital, en 1er. Simposio Síntesis Nacional y Regional de la Dinámica del Carbono del Suelo, Zacatecas, México, Sociedad Mexicana de la Ciencia del Suelo.

Sánchez-González, A., Chapela, M., Germán, E., Fuentes-García, R., del Río-Portilla, F., Siebe, C., 2017, Changes in quality and quantity of soil organic matter stocks resulting from wastewater irrigation in formely forested land. Geoderma, 306, 99-107.

Secretaría de Medio Ambiente y Recursos Naturales (SEMARNAT-CP-INEGI), 1999, Mapa de suelos dominantes de México, México.

Seol, Y., Lee, L.S., 2000, Effect of dissolved organic matter in treated effluents on sorption of atrazine and prometryn by soils: Soil Science Society of America Journal, 64, 1976-1983.

Sharom, M.S., Stephenson, G.R., 1976, Behavior and fate of metribuzin in eight Ontario soils: Weed Science, 24(2), 153-160.

Shea, P.J., 1989, Role of humified organic matter in herbicide adsorption: Weed Technology, 3, 190-197.

Sheng, G., Johnston, C.T., Teppen, B.J., Boyd, S.A., 2001, Potential contributions of smectite clays and organic matter to pesticide retention in soils: Journal of Agricultural and Food Chemistry, 49, 2899-2907.

Shoji, S., Nanzyo, M., Dahlgren, R.A., Quantin, P., 1996, Evaluation and proposed revisions of criteria for Andosols in the world reference base for soil resources: Soil Science, 161(9), 604-614.
Shukla, A., Devine, M.D., 2008, Basis of crop selectivity and weed resistance to triazine herbicides, en LeBaron, H.M., McFarland, J.E., Burnside, O.C. (eds.), The Triazine Herbicides, 50 Years Revolutionizing Agriculture, 1st. ed., San Diego, CA, Elsevier, 111-118.

Siebe, C., 1994, Akkumulation, Mobilitätund Verfügbarkeit von Schwermetallen in langjährigmit Abwasserbewässert en Böden Zentral mexikos. Hohenheimer Bodenkundliche Hefte 17, Stuttgart, Institut für Bodenkunde und Standortslehre Universität Hohenheim.

Siebe, C., Chapela-Lara, M., Cayetano-Salazar, M., Prado, B., Siemens, J., 2016, Effects of more than 100 years of irrigation with Mexico City's wastewater in the Mezquital Valley, en Hettiarachchi, H., Ardakanian, R. (eds.), Safe use of wastewater in agriculture: Good practice examples: UNU-FLORES, 121-138.

Simunek, J., Sejna, M., van Genuchten, M.Th., 1999, The HYDRUS-2D software package for simulating the two-dimensional movement of water, heat, and multiple solutes in variably-saturated media: Version 2.0, IGWCU-TPS - 53 International Ground Water Modelling Center, Colorado School of Mines, Golden, Colorado.

Siregar, A., Kleber, M., Mikutta, R., Jahn, R., 2005, Sodium hypochlorite oxidation reduces soil organic matter concentrations without affecting inorganic soil constituents: European Journal of Soil Science, 56, 481-490.

Spark, K.M., Swift, R.S., 2002, Effect of soil composition and dissolved organic matter on pesticide sorption: Science of the Total Environment, 298, 147-161.

Stevenson, FJ., 1994, Organic matter reactions involving pesticides in soil, in Humus chemistry: Genesis, Composition, Reactions. 2nd. Ed., NY, John Wiley and Sons, Inc., 454-471. 
Tafoya, A., 2008, Biodegradación del herbicida triazínico, atrazina, por una comunidad bacteriana, seleccionada en quimiostato, inmovilizada en reactores de lecho empacado, Instituto Politécnico Nacional, Escuela Nacional de Ciencias Biológicas. Tesis Doctoral.

Thomsen, M., Dobel, S., Lassen, P., Carlsen, L., Bugel Mogensen, B., Erik Hansen, P., 2002, Reverse quantitative structure-activity relationship for modelling the sorption of esfenvalerate to dissolved organic matter: a multivariate approach: Chemosphere, 49, 1317-1325.

Tomlin, G.D.S., 1997, The Pesticide Manual, 11 th ed., Farnham, United Kingdom, British Crop Protection Council.

Travis, M.J., Wiel-Shafran, A., Weisbrod, N., Adar, E., Gross, A., 2010, Greywater reuse for irrigation: Effect on soil properties: Science of the Total Environment, 408, 2501-2508.

United States Environmental Protection Agency (USEPA), 2003, Interim registration eligibility decision for atrazine, Washington, D.C. 285 p.

Urbanek, E., Horn, R., 2006, Changes in soil organic matter, bulk density and tensile strength of aggregates after percolation in conservation and conventional tilled soils: International Agrophysics, 20, 245-255.
Van-Ranst, E., Utami, S.R., Schamshuddin, J., 2002, Andisols on volcanic ash from Java Island, Indonesia: Physico-chemical properties and classification: Soil Science, 167(1), 68-79.

Viramontes, D., Rojas, F., Duwig, C., Esteves, M., 2006, Caracterización hidroerosiva de suelos de tipo Andosol e importancia de este recurso en el Estado de Michoacán, en GutiérrezLópez, A., Ramírez-Orozco, A.I., Sánchez Ramos, F. (eds.), Las Ciencias del Agua en Morelia, aplicaciones frente a los retos del siglo, vol. XXI: Morelos / Morelia, México, Ediciones IMTA-UMSNH, 149-161.

Wall, P.C., 2006, Facilitating the widespread adoption of conservation agriculture and other resource conserving technologies (RCT's): some difficult issues: Science Week Extended Abstracts, GIMMYT Headquarters, El Batán, México, 23-26, 61-64.

Wang, X., Guo, X., Yang, Y., Tao, S., Xing, B., 2011, Sorption mechanisms of phenanthrene, lindane, and atrazine with various humic acid fractions from a single soil sample: Environmental Science and Technology, 45, 2124-2130. 